Mon. Not. R. Astron. Soc. 000, 000-000 (0000) Printed 29 August $2017 \quad$ (MN LATEX style file v2.2)

\title{
Kinematics of OB-associations in Gaia epoch
}

\author{
A.M.Melnik ${ }^{\star}$ and A.K. Dambis \\ Sternberg Astronomical Institute, Lomonosov Moscow State University, Universitetskii pr. 13, Moscow, 119991 Russia
}

Accepted 2017 August 23. Received 2017 December 23; in original form 2017 April 11

\begin{abstract}
We use stellar proper motions from the TGAS catalog to study the kinematics of OBassociations. The TGAS proper motions of OB-associations generally agree well with the Hipparcos proper motions. The parameters of the Galactic rotation curve obtained with TGAS and Hipparcos proper motions agree within the errors. The average onedimensional velocity dispersion inside 18 OB-associations with more than 10 TGAS stars is $\sigma_{v}=3.9 \mathrm{~km} \mathrm{~s}^{-1}$, which is considerably smaller, by a factor of 0.4 , than the velocity dispersions derived from Hipparcos data. The effective contribution from orbital motions of binary OB-stars into the velocity dispersion $\sigma_{v}$ inside OB-associations is $\sigma_{b}=1.2 \mathrm{~km} \mathrm{~s}^{-1}$. The median virial and stellar masses of OB-associations are equal to $7.110^{5}$ and $9.010^{3} \mathrm{M}_{\odot}$, respectively. Thus OB-associations must be unbound objects provided they do not include a lot of dense gas. The median star-formation efficiency is $\epsilon=2.1$ percent. Nearly one third of stars of OB-associations must lie outside their tidal radius. We found that the Per OB1 and Car OB1 associations are expanding with the expansion started in a small region of $11-27$ pc 7-10 Myr ago. The average expansion velocity is $6.3 \mathrm{~km} \mathrm{~s}^{-1}$.
\end{abstract}

Key words: Galaxy: kinematics and dynamics - open clusters and associations

\section{INTRODUCTION}

Galactic astronomy is on the threshold of a new era of high precision proper motions with the recent first Gaia data release (Gaia DR1, Gaia Collaboration et al. 2016b) from the European Space Agency (ESA). Gaia DR1 contains the Tycho-Gaia Astrometric Solution (TGAS, Michalik, Lindegren \& Hobbs 2015; Lindegren et al. 2016) which provides positions, parallaxes and proper motions for about 2 million stars using the $24 \mathrm{yr}$ time difference between Hipparcos (ESA 1997; Hog et al. 2000) and Gaia (Gaia Collaboration et al. 2016a) observations.

The term 'OB-association' was first introduced by Ambartsumian (1949). The sizes of OB-associations differ from $10 \mathrm{pc}$ (Cyg OB2) to $500 \mathrm{pc}$ (Cep OB1), although the sky-plane sizes of 90 percent of them do not exceed 200 pc (Blaha \& Humphreys 1989). They often contain young clusters in their centres (Garmany \& Stencel 1992) and sometimes have several centres of concentration (Mel'nik \& Efremov 1995). The catalog compiled by Blaha \& Humphrevs (1989) includes 91 OB-associations located within $3.5 \mathrm{kpc}$ from the Sun. This catalog may also include several young open clusters because it is difficult to make a clear distinction between OB-associations and young open clusters. Though, OB-associations have, on average, larger sizes: 80 percent of them are larger than $d>20$ pc.

^ E-mail: anna@sai.msu.ru
Moreover, OB-associations are less centrally concentrated than young open clusters.

There are several partitions of high-luminosity stars (OB-stars and red supergiants) into OB-associations (Humphreys \& McElroy 1984; Blaha \& Humphreys 1989; Garmany \& Stencel 1992; Mel'nik \& Efremov 1995). Garmany \& Stencel (1992) divided young stars into OB-associations in the sector of Galactic longitudes 55$150^{\circ}$. Mel'nik \& Efremov (1995) used cluster analysis method to identify the densest and most compact parts of OB-associations, but these groups include only few stars with known kinematical data. Both lists of OBassociations are based on photometric data obtained by Blaha \& Humphrevs (1989). Here we consider the partition of Blaha \& Humphrevs (1989) as the most universal.

The catalog by Blaha \& Humphrevs (1989) includes the massive end of the stellar population of OB-associations. In some other studies (for example, de Zeeuw et al. 1999), the authors are concerned with the entire stellar population and also consider smaller scales, such as subgroups within larger OB-associations.

OB-associations are supposed to form in giant molecular clouds Elmegreen 1983; Zinnecker \& Yorke 2007, and references therein). The diameters and masses of giant molecular clouds lie in the range $10-80 \mathrm{pc}$ and $10^{5}-210^{6}$ $\mathrm{M}_{\odot}$ (Sanders, Scoville \& Solomon 1985), respectively. The catalog of Galactic giant molecular clouds is expected to be essentially complete at $M>310^{5} \mathrm{M}_{\odot}$ (Solomon et al. 1987; 
Solomon \& Rivolo 1989). There is a lot of evidence that giant molecular clouds are close to virial equilibrium (Larson 1981; Krumholz. Matzner \& McKee 2006).

The efficiency of star formation, $\epsilon$, determined as the ratio of the stellar mass of OB-association to the gaseous mass of its parent giant molecular clouds usually lies in the range of $0.1-10$ percent (Mvers et al. 1986; Evans et al. 2009; Garcia et al. 2014). The low efficiency of star formation can be explained by two different ways: through destruction of molecular clouds by the radiation of high luminosity stars (for example, Elmegreen 1983; Franco, Shore \& Tenorio-Tagle 1994; Colin, Vazquez-Semadeni \& Gomez 2013) and through supersonic turbulence and magnetic fields preventing global collapse of the cloud (Mac Low \& Klessen 2004; McKee \& Ostriker 2007).

Blaauw (1964) found the expansion of OB-associations by analyzing ground-based proper motions of their member stars. Brown, Dekker \& de Zeeuw (1997) simulated the expansion of OB-associations to check methods for deriving their kinematic age - the time in the past when the OBassociation had minimal size. Madsen. Dravins \& Lindegren (2002) find some evidence for expansion in Sco OB2 by comparing spectroscopic radial velocities with values derived from the hypothesis that members of the OB-association share the same velocity vector. Although the estimated expansion velocities of OB-associations decrease with growing accuracy of proper motions, the expansion of these systems is expected in many scenarios of star formation.

The mass loss in a gas cloud due to thermal pressure of HII regions (McKee 1989; Kim, Kim \& Ostriker 2016) can make the newly formed stellar group unbound. If mass is ejected from the system within a time comparable to the crossing time, then the system becomes unbound after 50 percent mass loss (Hills 1980). However, more accurate treatment of relaxation processes shows that the system can form an expanding OB-association with a bound cluster in the centre (Tutukov 1978; Kroupa, Aarseth \& Hurley 2001; Boily \& Kroupa 2003a b; Vine \& Bonnell 2003; Baumgardt \& Kroupa 2007).

We study the kinematics of OB-associations using the stellar proper motions from the TGAS catalog (Michalik, Lindegren \& Hobbs 2015). The high precision of TGAS proper motions makes it possible to estimate the virial masses of OB-associations corresponding to the masses of their parent molecular clouds, and examine the expansion of OB-associations at present time.

In section 2 we investigate the motions of OBassociations as single entities: we derive the parameters of the Galactic rotation curve, study the residual velocities and the motion along the Z-axis. In section 3 we study internal properties of OB-associations: we determine their velocity dispersions, virial and stellar masses, estimate the star formation efficiency and tidal radii. We consider the expansion of OB-associations in Section 4 and formulate the main conclusions in Section 5.

\section{MOTIONS OF OB-ASSOCIATIONS IN THE GALAXY}

\subsection{Kinematic data of stars in OB-associations}

We calculate the median proper motions of OBassociations using TGAS proper motions of individual stars (Michalik, Lindegren \& Hobbs 2015). On the whole, OBassociations identified by Blaha \& Humphreys (1989) include 500 stars with known TGAS proper motions. For comparison, Hipparcos catalog contains 774 star members of OB-associations. The lack of TGAS proper motions mostly concerns nearby OB-associations, which contain bright stars $\left(m_{v}<7^{m}\right)$. Determining astrometric parameters of bright stars requires additional calibrations and for this reason TGAS lacks many bright stars from nearby OB-associations. For example, OB-association Ori OB1 includes 59 stars with known Hipparcos proper motions and only three stars with TGAS data. We therefore consider only proper motions of stars in OB-associations and do not use their parallaxes. It is nearby stars with the most precise parallaxes that are most important for the study of the distance scale.

The mass measurements of proper motions of blue stars became available due to the inclusion of a list of OB-stars into the Hipparcos input catalog (de Zeeuw et al. 1999) providing very accurate first-epoch positions and hence very small TGAS proper-motion errors for OB-association members. The average error of TGAS proper motions for OB-

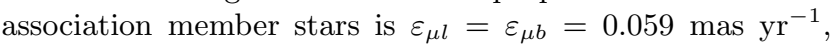
which is nearly 15 times smaller than the average error of Hipparcos proper motions, 0.916 mas $\mathrm{yr}^{-1}$, for OBassociation stars.

Table 1 presents the average Galactic coordinates of OB-associations, $l$ and $b$, the average heliocentric distances to the associations, $r$, the median proper motions in l- and b-directions, $\mu_{l}$ and $\mu_{b}$, the dispersion of proper motions $\sigma_{\mu l}$ and $\sigma_{\mu b}$ and the number of stars in OB-associations with known TGAS proper motions, $n_{\mu}$. To complete the kinematic data we also give the median line-of sight velocities, $V_{r}$, the dispersion of the line-of-sight velocities $\sigma_{v r}$ and the number of stars with known line-of-sight velocities, $n_{v r}$, taken from the catalog by Barbier-Brossat \& Figon (2000). Table 1 also gives the total number of stars in an OB-association with known photometric measurements, $N_{t}$, used by Blaha \& Humphreys (1989) to determine distances for OB-associations, $r_{B H}$.

Note that the errors of the listed proper motions and line-of-sight velocities of OB-associations, $\varepsilon_{\mu l}, \varepsilon_{\mu b}$ and $\varepsilon_{v r}$, are calculated in the following way:

$$
\begin{aligned}
& \varepsilon_{\mu l}=\frac{\sigma_{\mu l}}{\sqrt{n_{\mu}}}, \\
& \varepsilon_{\mu b}=\frac{\sigma_{\mu b}}{\sqrt{n_{\mu}}}, \\
& \varepsilon_{v r}=\frac{\sigma_{v r}}{\sqrt{n_{v r}}} .
\end{aligned}
$$

The method of deriving the robust estimates of the dispersion of $\mu_{l}, \mu_{b}$ and $V_{r}$ inside the OB-association is also discussed in section 3.1 .

Table 2, which is available in the online version of the paper, gives the spectral, photometric and kinematic data of stars in OB-associations. It presents the name of a star, the name of the OB-association to which it is assigned by 
Blaha \& Humphreys (1989), spectral type of the star, code of its luminosity class $c_{L}: 2-\mathrm{Ia}, 4-\mathrm{Iab}, 6-\mathrm{Ib}, 8-\mathrm{II}, 10-\mathrm{III}$, $12-\mathrm{IV}, 14-\mathrm{V}$, where the corresponding odd numbers (1, 3, $\ldots, 13)$ reflect the uncertainty in its determination. Table 2 also shows Galactic coordinates $l$ and $b$ of a star, and the heliocentric distance $r$ to OB-association, which it is assigned to. We also present the line-of-sight velocity of a star $V_{r}$ and its error $\varepsilon_{v r}$ taken from the catalog Barbier-Brossat \& Figon (2000). Note that we use only the individual stellar velocities $V_{r}$ determined with the error $\varepsilon_{v r} \leqslant 10 \mathrm{~km} \mathrm{~s}^{-1}$ to derive the median values of $V_{r}$ and velocity dispersions $\sigma_{v r}$ in OB-association. For the Hipparcos stars we present their Hipparcos number $n_{\text {Hip }}$, TGAS proper motions, $\mu_{l}$ and $\mu_{b}$, if avalable, and their errors, $\varepsilon_{\mu l}$ and $\varepsilon_{\mu b}$. If a Hipparcos star is absent in the TGAS catalog, then we give its Hipparcos proper motions and their errors; flag $\mathrm{F}$ indicates the source of proper motions: 'G' means TGAS and 'H' - Hipparcos. Table 2 also represents color indexes $B-V$ and $U-B$, apparent and absolute magnitudes, $m_{V}$ and $M_{V}$, and the $V$-band extinction, $A_{V}$, that are adopted from the catalog by Blaha \& Humphreys (1989).

We reduced the heliocentric distances to OBassociations derived by Blaha \& Humphrevs (1989), $r_{B H}$, to the short distance scale, $r=0.8 \cdot r_{B H}$ (Sitnik \& Mel'nik 1996; Dambis, Mel'nik \& Rastorguev 2001; Mel'nik \& Dambis 2009). We also correct the absolute stellar magnitudes obtained by Blaha \& Humphrevs (1989), $M_{V(B H)}$, to bring them onto the short distance scale $M_{V}=M_{V(B H)}+\Delta m$, where $\Delta m=-5 \log 0.8=0.485^{m}$.

\subsection{Comparison TGAS and Hipparcos proper motions of OB-associations}

We compare the median proper motions $\mu_{l}$ and $\mu_{b}$ of OBassociations derived from data of Hipparcos and TGAS catalogs. Only 56 OB-associations containing at least two stars with known proper motions are considered. Figure 1 shows the correlation between the Hipparcos and TGAS proper motions. The vast interval spanned by $\mu_{l}$ values, $[-10,+6]$ mas $\mathrm{yr}^{-1}$, is due to differential Galactic rotation. The distribution of proper-motion components $\mu_{b}$ is more compact, reflecting small motions perpendicular to the Galactic plane. Two associations with large negative $\mu_{b}$ (Coll 140 and Vela OB2) are located close to the Sun, $r=0.3$ and $0.4 \mathrm{kpc}$, so their large negative proper motions translate into the velocities of only -7 and $-9 \mathrm{~km} \mathrm{~s}^{-1}$. The $\mu_{b}$ proper-motion components are mostly negative reflecting the positive velocity of the solar motion perpendicular to the Galactic plane, $w_{0}$, which is equal to about $+7 \mathrm{~km} \mathrm{~s}^{-1}$. The rms difference of TGAS and Hipparcos proper motions is the same for l- and

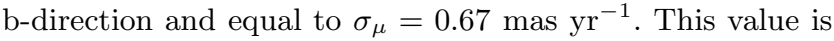
comparable to the average error of proper motions of OBstars in the Hipparcos catalog, 0.916 mas $\mathrm{yr}^{-1}$. Thus the mean difference of velocities calculated at the mean heliocentric distance of OB-associations $(r=1.5 \mathrm{kpc})$ is $\Delta v=4.7$ $\mathrm{km} \mathrm{s}^{-1}$.

\subsection{Galactic rotation curve}

We determine the parameters of the Galactic rotation curve assuming to a first approximation that OB-associations move in circular orbits in accordance with Galactic differential rotation. The method of the determination of the parameters of the rotation curve is described in detail in Mel'nik \& Dambis (2009).

Briefly, we expand the angular rotation velocity $\Omega(R)$ into a power series in $R-R_{0}$ to the second order and determine values of three parameters of the rotation curve: the angular velocity at the solar distance $\Omega_{0}$ as well as the first and second derivatives of $\Omega(R)$ taken at the solar distance, $\Omega_{0}^{\prime}$ and $\Omega_{0}^{\prime \prime}$, respectively. We also determine the solar velocity components $u_{0}$ and $v_{0}$ with respect to the centroid of OB-associations in the direction toward the Galactic centre and in the sense of Galactic rotation, correspondingly.

We use two samples of OB-associations (denoted as samples 1 and 2). Sample 1 includes objects whose median line-of-sight velocities $V_{r}$ or proper motions $\mu_{l}$ were derived from the data for at least two member stars, whereas sample 2 is based on the velocities and proper motions obtained from the data for at least five member stars. Sample 1 provides 70 and 56 equations for $V_{r}$ and $\mu_{l}$, respectively. Sample 2 gives 50 equations for $V_{r}$ and 32 equations for $\mu_{l}$. We solve the equations for the line-of-sight velocities and proper motions jointly and use weight factors to allow for observational errors and "cosmic" velocity dispersion (see also Dambis, Mel'nik \& Rastorguev 1995; Mel'nik, Dambis \& Rastorguev 1999, 2001). We use standard least square method (Press at al. 1987) to solve the systems of 126 (sample 1) and 82 (sample 2) equations, which are linear in the parameters $\Omega_{0}, \Omega_{0}^{\prime}, \Omega_{0}^{\prime \prime}, u_{0}$, and $v_{0}$.

We adopt a solar Galactocentric distance of $R_{0}=7.5 \mathrm{kpc}$ (Rastorguev et al. 1994; $\quad$ Dambis, Mel'nik \& Rastorguev 1995; Glushkova et al. 1998; Nikiforov 2004; Feast et al. 2008; Groenewegen, Udalski \& Bono 2008; Reid at al. 2009b; Dambis et al. 2013; Francis \& Anderson 2014; Boehle et al. 2016; Branham 2017). Note that the particular choice of $R_{0}$ in the range $7-9 \mathrm{kpc}$ has practically no effect on the analysis of the space distribution and kinematics of stars located within $3 \mathrm{kpc}$ from the Sun.

Table 3 lists the parameters of the Galactic rotation curve, $\Omega_{0}, \Omega_{0}^{\prime}$ and $\Omega_{0}^{\prime \prime}$, and the solar motion toward the apex, $u_{0}$ and $v_{0}$, determined for samples 1 and 2 . It also lists the inferred Oort constant $A=-0.5 R_{0} \Omega_{0}^{\prime}$, the standard deviation of the velocities from the rotation curve $\sigma_{0}$, and the number of conditional equations $N_{e q}$.

We can see that the parameters of the Galactic rotation curve and solar motion to the apex derived for samples 1 and 2 agree within the errors. They also agree well with the parameters obtained for Hipparcos data (see Table 2 in Mel'nik \& Dambis 2009). The greatest difference between the parameters derived from TGAS and Hipparcos proper motions concerns the case of the second derivative of the angular velocity $\Omega(R)$ taken at the solar distance, $\Omega_{0}^{\prime \prime}: \Omega_{0}^{\prime \prime}=1.11 \pm 0.22$ and $1.35 \pm 0.20 \mathrm{~km} \mathrm{~s}^{-1} \mathrm{kpc}^{-3}$, respectively. Note that both TGAS and Hipparcos data yield large values for the angular velocity at the solar distance $\Omega_{0}$ equal to $31.08 \pm 0.86$ and $30.55 \pm 0.87 \mathrm{~km} \mathrm{~s}^{-1} \mathrm{kpc}^{-1}$, respectively. The $\Omega_{0}=31.08 \mathrm{~km} \mathrm{~s}^{-1} \mathrm{kpc}^{-1}$ estimate translates into a solar azimuthal velocity of $\Theta=233 \mathrm{~km} \mathrm{~s}^{-1}$. A large $\Omega_{0}$ value of $30-31 \mathrm{~km} \mathrm{~s}^{-1} \mathrm{kpc}^{-1}$ is also inferred from an analysis of the motions of young open clusters with ages less than 100 Myr (Melnik et al. 2016) and Galactic maser 
Table 1. Line-of-sight velocities and TGAS proper motions of OB-associations

\begin{tabular}{|c|c|c|c|c|c|c|c|c|c|c|c|c|}
\hline Name & $\begin{array}{c}l \\
\text { deg. }\end{array}$ & $\begin{array}{c}b \\
\text { deg. }\end{array}$ & $\begin{array}{c}r \\
\mathrm{kpc}\end{array}$ & $N_{t}$ & $\begin{array}{c}V_{r} \\
\mathrm{~km} \mathrm{~s}^{-1}\end{array}$ & $\begin{array}{c}\sigma_{v r} \\
\mathrm{~km} \mathrm{~s}^{-1}\end{array}$ & $n_{v r}$ & $\begin{array}{c}\mu_{l} \\
\text { mas } \mathrm{yr}^{-1}\end{array}$ & 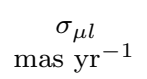 & $\begin{array}{c}\mu_{b} \\
\text { mas } \mathrm{yr}^{-1}\end{array}$ & 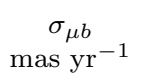 & $n_{\mu}$ \\
\hline SGR OB5 & 0.02 & -1.19 & 2.42 & 31 & -15.0 & 19.0 & 2 & -0.35 & & -2.39 & & 1 \\
\hline SGR OB1 & 7.55 & -0.77 & 1.26 & 65 & -10.0 & 12.1 & 37 & -1.10 & 0.22 & -1.30 & 0.78 & 13 \\
\hline SGR OB7 & 10.73 & -1.57 & 1.39 & 4 & -6.1 & 17.2 & 3 & & & & & 0 \\
\hline SGR OB4 & 12.11 & -0.96 & 1.92 & 15 & 3.5 & 10.7 & 9 & -0.24 & 0.79 & -1.41 & 0.34 & 2 \\
\hline SGR OB6 & 14.19 & 1.28 & 1.60 & 5 & -7.3 & 0.1 & 4 & & & & & 0 \\
\hline SER OB1 & 16.72 & 0.07 & 1.53 & 43 & -5.0 & 20.0 & 17 & -1.10 & 0.63 & -0.87 & 0.34 & 6 \\
\hline SCT OB3 & 17.30 & -0.73 & 1.33 & 10 & 3.3 & 17.0 & 8 & -2.51 & 0.61 & -1.07 & 0.64 & 3 \\
\hline SER OB2 & 18.21 & 1.63 & 1.60 & 18 & -4.0 & 14.5 & 7 & -0.98 & 0.11 & -0.16 & 0.08 & 2 \\
\hline SCT OB2 & 23.18 & -0.54 & 0.80 & 13 & -11.0 & 20.0 & 6 & -0.51 & 0.34 & -0.74 & 0.04 & 4 \\
\hline TR 35 & 28.03 & -0.46 & 2.01 & 9 & 31.0 & & 1 & -3.05 & & -0.93 & & 1 \\
\hline COLL 359 & 29.79 & 12.63 & 0.16 & 1 & & & 0 & & & & & 0 \\
\hline VUL OB1 & 60.35 & 0.03 & 1.60 & 28 & 3.1 & 14.7 & 9 & -4.98 & 1.10 & -0.86 & 0.52 & 8 \\
\hline VUL OB4 & 60.63 & -1.22 & 0.80 & 9 & -2.9 & 7.5 & 3 & -5.10 & 0.21 & -1.74 & 0.65 & 2 \\
\hline CYG OB3 & 72.77 & 2.03 & 1.83 & 42 & -9.5 & 9.5 & 30 & -7.15 & 0.30 & -0.89 & 0.18 & 16 \\
\hline CYG OB1 & 75.84 & 1.12 & 1.46 & 71 & -13.5 & 10.5 & 34 & -6.35 & 0.54 & -0.58 & 0.36 & 12 \\
\hline CYG OB9 & 77.81 & 1.80 & 0.96 & 32 & -19.5 & 8.7 & 10 & -6.44 & 1.13 & -1.95 & 1.22 & 5 \\
\hline CYG OB8 & 77.91 & 3.36 & 1.83 & 21 & -21.0 & 11.0 & 9 & -6.27 & 0.43 & 0.53 & 1.60 & 10 \\
\hline CYG OB2 & 80.27 & 0.88 & 1.46 & 15 & & & 0 & -5.24 & & -0.80 & & 1 \\
\hline CYG OB4 & 82.69 & -7.48 & 0.80 & 2 & -4.9 & 1.1 & 2 & & & & & 0 \\
\hline CYG OB7 & 88.99 & 0.03 & 0.63 & 29 & -9.4 & 9.3 & 21 & -2.43 & 3.77 & -1.01 & 0.68 & 16 \\
\hline NGC 6991 & 87.58 & 1.42 & 1.39 & 1 & -15.0 & & 1 & & & & & 0 \\
\hline LAC OB1 & 96.71 & -17.70 & 0.48 & 2 & -13.6 & 4.2 & 2 & & & & & 0 \\
\hline CEP OB2 & 102.04 & 4.68 & 0.73 & 57 & -17.0 & 6.7 & 36 & -3.84 & 0.91 & -0.77 & 1.23 & 34 \\
\hline CEP OB1 & 104.20 & -0.94 & 2.78 & 58 & -58.2 & 7.4 & 17 & -4.46 & 0.69 & -0.67 & 0.36 & 20 \\
\hline NGC 7235 & 102.78 & 0.77 & 3.18 & 1 & & & 0 & & & & & 0 \\
\hline CEP OB5 & 108.50 & -2.69 & 1.67 & 6 & -48.7 & 29.2 & 2 & -4.17 & & -0.47 & & 1 \\
\hline CAS OB2 & 111.99 & -0.00 & 2.10 & 41 & -50.1 & 11.0 & 7 & -4.02 & 0.07 & -0.59 & 0.31 & 5 \\
\hline CEP OB3 & 110.42 & 2.56 & 0.70 & 26 & -22.9 & 3.9 & 18 & -2.13 & 0.51 & -1.71 & 0.40 & 13 \\
\hline CAS OB5 & 116.10 & -0.50 & 2.01 & 52 & -45.8 & 7.2 & 16 & -3.72 & 0.86 & -0.86 & 0.41 & 8 \\
\hline CEP OB4 & 118.21 & 5.25 & 0.66 & 7 & -24.0 & & 1 & -1.84 & 0.05 & -1.29 & 0.04 & 4 \\
\hline CAS OB4 & 120.05 & -0.30 & 2.30 & 27 & -37.0 & 8.6 & 7 & -2.50 & 0.17 & -0.92 & 0.13 & 6 \\
\hline CAS OB14 & 120.37 & 0.74 & 0.88 & 8 & -15.0 & 7.0 & 4 & -0.87 & 0.08 & -1.21 & 0.99 & 2 \\
\hline CAS OB7 & 122.98 & 1.22 & 2.01 & 39 & -50.0 & 1.0 & 4 & -2.47 & 0.23 & -0.47 & 0.24 & 7 \\
\hline CAS OB1 & 124.72 & -1.73 & 2.01 & 11 & -42.0 & 2.5 & 5 & -1.51 & 0.20 & -1.27 & 0.27 & 3 \\
\hline NGC 457 & 126.64 & -4.43 & 2.01 & 4 & -34.8 & 2.3 & 4 & -1.55 & 0.06 & -0.93 & 0.02 & 2 \\
\hline CAS OB8 & 129.16 & -1.06 & 2.30 & 43 & -34.6 & 9.9 & 14 & -1.00 & 0.10 & -0.61 & 0.15 & 8 \\
\hline PER OB1 & 134.70 & -3.16 & 1.83 & 165 & -43.2 & 7.0 & 80 & -0.19 & 0.58 & -1.23 & 0.36 & 58 \\
\hline CAS OB6 & 135.02 & 0.75 & 1.75 & 46 & -42.6 & 8.1 & 12 & -0.38 & 0.29 & -0.74 & 0.47 & 11 \\
\hline CAM OB1 & 141.08 & 0.89 & 0.80 & 50 & -11.0 & 9.4 & 30 & 0.13 & 1.25 & -1.27 & 0.92 & 26 \\
\hline CAM OB3 & 146.99 & 2.85 & 2.65 & 8 & -27.6 & 19.3 & 3 & -0.70 & & 0.35 & & 1 \\
\hline PER OB3 & 146.64 & -5.86 & 0.14 & 1 & -2.4 & & 1 & & & & & 0 \\
\hline PER OB2 & 160.24 & -16.55 & 0.32 & 7 & 21.2 & 4.5 & 7 & 5.60 & 0.15 & -1.16 & 3.89 & 3 \\
\hline AUR OB1 & 173.83 & 0.14 & 1.06 & 36 & -1.9 & 14.0 & 26 & 2.25 & 0.51 & -1.57 & 0.30 & 12 \\
\hline ORI OB1 & 206.94 & -17.71 & 0.40 & 68 & 25.4 & 7.9 & 62 & -0.03 & 2.28 & -0.30 & 0.59 & 3 \\
\hline AUR OB2 & 173.33 & -0.17 & 2.42 & 20 & -2.6 & 4.9 & 4 & 0.85 & 0.84 & -0.79 & 0.30 & 2 \\
\hline NGC 1893 & 173.60 & -1.70 & 2.90 & 10 & & & 0 & & & & & 0 \\
\hline NGC 2129 & 186.45 & -0.11 & 1.46 & 3 & 16.8 & 7.5 & 3 & 1.90 & & -0.91 & & 1 \\
\hline GEM OB1 & 188.98 & 2.22 & 1.21 & 40 & 16.0 & 5.0 & 18 & 1.53 & 0.39 & -0.56 & 0.31 & 6 \\
\hline MON OB1 & 202.10 & 1.08 & 0.58 & 7 & 23.4 & 13.0 & 7 & 0.27 & 0.32 & -1.62 & 0.18 & 4 \\
\hline MON OB2 & 207.46 & -1.65 & 1.21 & 32 & 22.3 & 12.5 & 26 & -1.10 & 0.22 & -1.31 & 0.47 & 10 \\
\hline MON OB3 & 217.65 & -0.44 & 2.42 & 4 & 27.0 & & 1 & & & & & 0 \\
\hline CMA OB1 & 224.58 & -1.56 & 1.06 & 17 & 34.3 & 16.2 & 8 & -2.93 & 0.78 & -1.93 & 1.15 & 7 \\
\hline NGC 2414 & 231.09 & 1.01 & 3.18 & 15 & 67.2 & & 1 & -1.94 & & -0.50 & & 1 \\
\hline COLL 121 & 238.45 & -8.41 & 0.55 & 13 & 29.6 & 7.0 & 10 & -4.73 & 1.77 & -1.53 & 0.37 & 3 \\
\hline NGC 2362 & 237.87 & -5.92 & 1.21 & 9 & 30.0 & 14.0 & 6 & & & & & 0 \\
\hline NGC 2367 & 235.65 & -3.84 & 2.20 & 5 & 37.0 & 9.0 & 4 & -3.11 & & -0.66 & & 1 \\
\hline NGC 2439 & 245.27 & -4.08 & 3.50 & 23 & 62.7 & & 1 & -4.27 & 0.43 & -0.54 & 0.18 & 10 \\
\hline PUP OB1 & 243.53 & 0.16 & 2.01 & 22 & 77.0 & & 1 & -3.66 & 0.11 & -0.94 & 1.64 & 3 \\
\hline PUP OB2 & 244.61 & 0.58 & 3.18 & 13 & & & 0 & & & & & 0 \\
\hline COLL 140 & 244.47 & -7.33 & 0.29 & 6 & 10.3 & 6.6 & 5 & -7.56 & 1.23 & -4.45 & 1.55 & 2 \\
\hline
\end{tabular}


Table 1. Line-of-sight velocities and TGAS proper motions of OB-associations (end)

\begin{tabular}{|c|c|c|c|c|c|c|c|c|c|c|c|c|}
\hline Name & $\begin{array}{c}l \\
\text { deg. }\end{array}$ & $\begin{array}{c}b \\
\text { deg. }\end{array}$ & $\begin{array}{c}r \\
\mathrm{kpc}\end{array}$ & $N_{t}$ & $\begin{array}{c}V_{r} \\
\mathrm{~km} \mathrm{~s}^{-1}\end{array}$ & $\begin{array}{c}\sigma_{v r} \\
\mathrm{~km} \mathrm{~s}^{-1}\end{array}$ & $n_{v r}$ & $\begin{array}{c}\mu_{l} \\
\operatorname{mas} \mathrm{yr}^{-1}\end{array}$ & $\begin{array}{c}\sigma_{\mu l} \\
\operatorname{mas} \mathrm{yr}^{-1}\end{array}$ & $\begin{array}{c}\mu_{b} \\
\operatorname{mas} \mathrm{yr}^{-1}\end{array}$ & 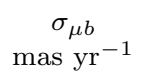 & $n_{\mu}$ \\
\hline PUP OB3 & 253.89 & -0.25 & 1.46 & 3 & & & 0 & & & & & 0 \\
\hline VELA OB2 & 262.08 & -8.52 & 0.39 & 13 & 24.0 & 9.7 & 13 & -9.73 & 0.32 & -4.88 & 2.97 & 2 \\
\hline VELA OB1 & 264.84 & -1.41 & 1.46 & 46 & 23.0 & 4.3 & 18 & -6.78 & 1.07 & -1.69 & 0.25 & 7 \\
\hline CAR OB1 & 286.45 & -0.46 & 2.01 & 126 & -5.0 & 8.2 & 39 & -8.08 & 0.74 & -0.81 & 0.24 & 15 \\
\hline TR 16 & 287.25 & -0.25 & 2.10 & 18 & -1.0 & 3.3 & 5 & -7.20 & 0.55 & -0.94 & 0.38 & 2 \\
\hline TR 14 & 287.37 & -0.47 & 2.78 & 5 & -10.0 & & 1 & & & & & 0 \\
\hline TR 15 & 287.65 & -0.42 & 3.04 & 2 & & & 0 & & & & & 0 \\
\hline COLL 228 & 287.57 & -0.98 & 2.01 & 15 & -13.0 & 9.0 & 9 & -6.92 & & -1.64 & & 1 \\
\hline CAR OB2 & 290.39 & 0.12 & 1.79 & 59 & -8.2 & 8.5 & 22 & -6.62 & 0.23 & -1.09 & 0.27 & 10 \\
\hline NGC 3576 & 291.33 & -0.61 & 2.53 & 5 & -17.0 & 9.0 & 2 & & & & & 0 \\
\hline CRU OB1 & 294.87 & -1.06 & 2.01 & 76 & -5.3 & 8.9 & 33 & -6.28 & 0.32 & -0.88 & 0.21 & 17 \\
\hline NGC 3766 & 294.11 & -0.02 & 1.53 & 12 & -15.6 & 0.7 & 2 & -6.76 & 0.01 & -1.10 & 0.09 & 2 \\
\hline CEN OB1 & 304.14 & 1.44 & 1.92 & 103 & -19.0 & 14.5 & 32 & -4.89 & 0.57 & -1.01 & 0.21 & 28 \\
\hline HOGG 16 & 307.51 & 1.39 & 1.46 & 5 & -35.0 & 8.5 & 3 & -3.70 & 0.01 & -1.09 & 0.24 & 2 \\
\hline R 80 & 309.37 & -0.41 & 2.90 & 2 & -38.2 & & 1 & & & & & 0 \\
\hline NGC 5606 & 314.87 & 0.99 & 1.53 & 5 & -37.8 & 1.8 & 3 & -5.60 & 0.04 & -0.85 & 0.01 & 2 \\
\hline CIR OB1 & 315.47 & -2.76 & 2.01 & 4 & & & 0 & & & & & 0 \\
\hline PIS 20 & 320.39 & -1.49 & 3.18 & 6 & -49.0 & & 1 & -4.98 & & -0.20 & & 1 \\
\hline NOR OB1 & 328.05 & -0.92 & 2.78 & 8 & -35.6 & 6.5 & 6 & -4.11 & & -0.69 & & 1 \\
\hline NGC 6067 & 329.71 & -2.18 & 1.67 & 9 & -40.0 & 2.6 & 8 & -3.18 & & -0.57 & & 1 \\
\hline R 103 & 332.34 & -0.75 & 3.18 & 34 & -48.0 & 26.0 & 11 & -5.04 & 1.38 & -1.52 & 0.73 & 3 \\
\hline R 105 & 333.08 & 1.90 & 1.26 & 4 & -31.0 & 7.0 & 4 & -3.04 & & 0.07 & & 1 \\
\hline ARA OB1B & 337.95 & -0.85 & 2.78 & 21 & -34.7 & 10.3 & 9 & -2.63 & 0.64 & -1.20 & 0.43 & 6 \\
\hline ARA OB1A & 337.69 & -0.92 & 1.10 & 53 & -36.3 & 20.6 & 8 & -2.05 & 0.33 & -2.59 & 0.12 & 5 \\
\hline NGC 6204 & 338.31 & -1.15 & 2.20 & 14 & -51.0 & 5.8 & 5 & & & & & 0 \\
\hline SCO OB1 & 343.71 & 1.36 & 1.53 & 76 & -28.8 & 17.5 & 28 & -2.26 & 0.41 & -0.56 & 0.32 & $\varepsilon$ \\
\hline SCO OB2 & 351.31 & 19.02 & 0.13 & 10 & -4.1 & 2.3 & 10 & & & & & 0 \\
\hline HD 156154 & 351.30 & 1.41 & 2.10 & 4 & -4.0 & 8.5 & 3 & -1.05 & 0.10 & -0.54 & 0.09 & 2 \\
\hline SCO OB4 & 352.64 & 3.23 & 0.96 & 11 & 3.0 & 6.3 & 7 & -1.34 & 0.42 & -2.70 & 0.29 & 3 \\
\hline TR 27 & 355.06 & -0.73 & 0.88 & 11 & -15.8 & & 1 & & & & & 0 \\
\hline M 6 & 356.75 & -0.87 & 0.37 & 1 & -6.4 & & 1 & & & & & 0 \\
\hline
\end{tabular}

Table 2. Spectral, photometric and kinematic data for stars in OB-associations (available online only)
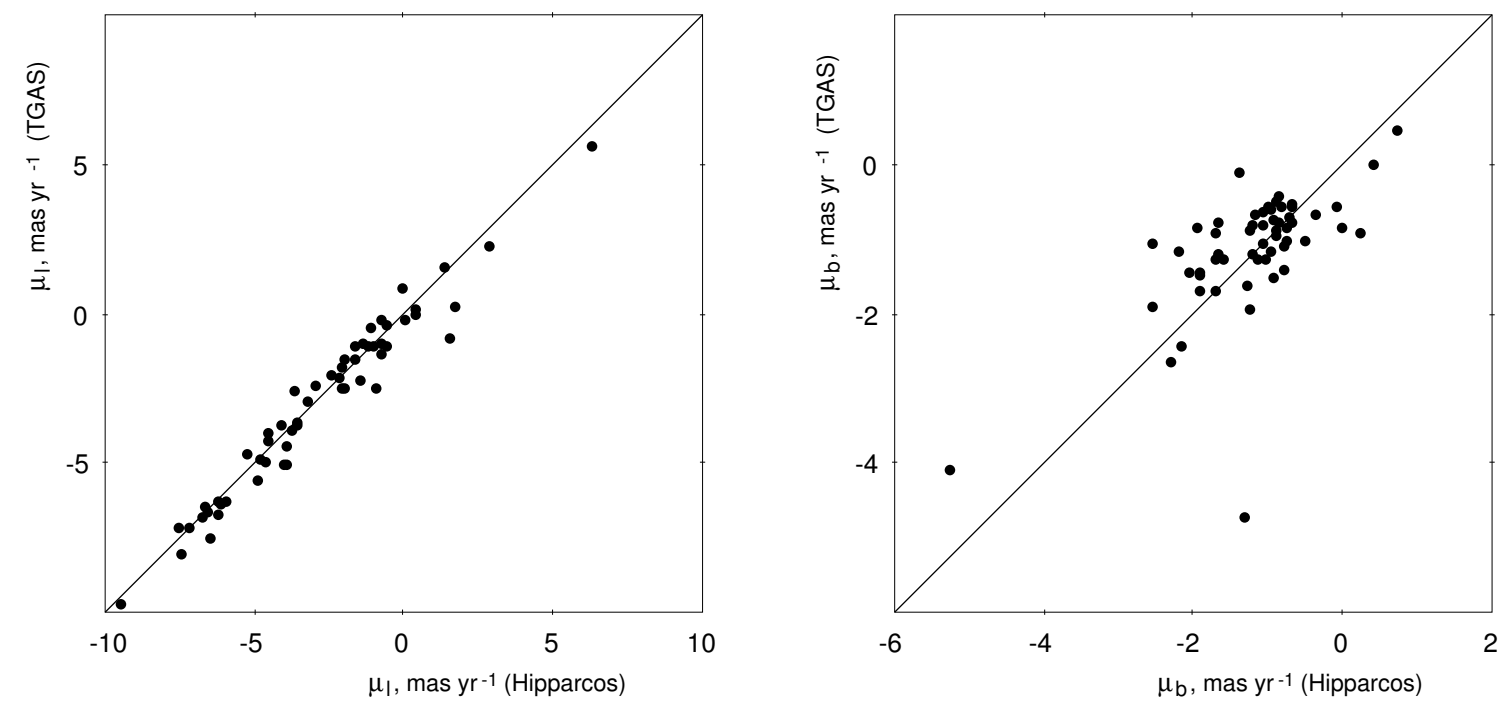

Figure 1. Comparison of proper motions $\mu_{l}$ and $\mu_{b}$ of OB-associations derived from data of Hipparcos and TGAS catalogs. Only 56 OB-associations containing at least two stars with known proper motions are considered. Note that the left and right images have different scales. The rms deviation of TGAS and Hipparcos proper motions is the same for l- and b-direction and equal to $\sigma_{\mu}=0.67 \mathrm{mas}^{\mathrm{yr}} \mathrm{r}^{-1}$. 


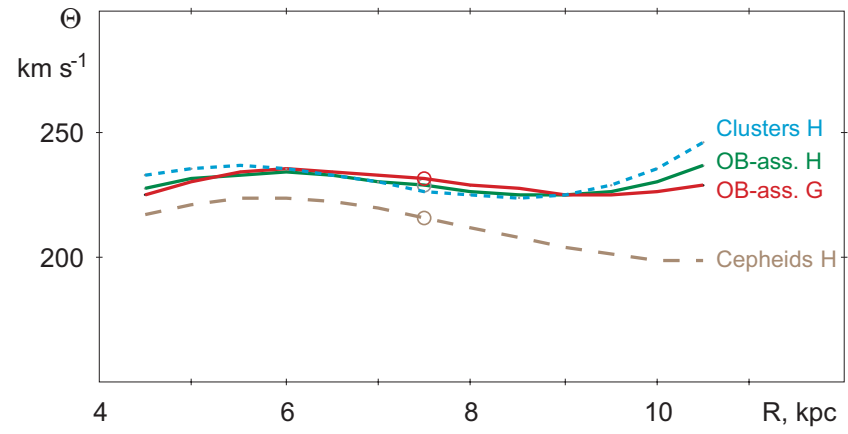

Figure 2. Galactic rotation curve obtained for different young objects: OB-associations with TGAS (sample 1) and Hipparcos data, young open clusters and classical Cepheids. The rotation curves based on TGAS and Hipparcos proper motions are labeled by the "G" and "H" letters, respectively. The circle shows the position of the Sun. We can see that the rotation curves obtained for OB-associations with TGAS and Hipparcos proper motions agree well with each other.

sources (Reid at al. 2009a; Bobylev \& Baikova 2012). However, Hipparcos proper motions of classical Cepheids yield a slightly smaller value of $\Omega_{0}, 28.8 \pm 0.8$ (Mel'nik et al. 2015).

Figure 2 shows the Galactic rotation curve obtained for different young objects: OB-associations with TGAS (sample 1) and Hipparcos (Mel'nik \& Dambis 2009) data, young open clusters (Melnik et al. 2016) and classical Cepheids (Mel'nik et al. 2015). We can see that the rotation curves calculated for OB-associations with TGAS and Hipparcos proper motions are in good agreement.

\subsection{Residual velocities}

The residual velocities are determined as the differences between the observed heliocentric velocities and the velocities due to the inferred rotation curve and the solar motion toward the apex $\left(V_{\text {res }}=V_{\text {obs }}-V_{\text {rot }}-V_{a p}\right)$. The residual velocities characterize non-circular motions in the Galactic disc. We consider the residual velocities in the direction of the Galactic radius-vector, $V_{R}$, and in the azimuthal direction, $V_{T}$. The velocity components $V_{R}$ and $V_{T}$ are positive when directed away from the Galactic centre and in the direction of the Galactic rotation, correspondingly.

Figure 3 shows the distribution of residual velocities of OB-associations in the Galactic plane derived with TGAS proper motions. It also shows the positions of the Sagittarius, Scorpio, Carina, Cygnus, Local System, and Perseus stellar-gas complexes from the list by Efremov \& Sitnik (1988). We can see that in some complexes the residual velocities $V_{R}$ and $V_{T}$ have a preferred direction. In the Perseus complex the average residual velocity components are $V_{R}=-5.5 \pm 2.3$ and $V_{T}=-4.7 \pm 1.6$, whereas in the Sagittarius complex they are equal to $V_{R}=+7.9 \pm 2.5$ and $V_{T}=-1.0 \pm 2.1 \mathrm{~km} \mathrm{~s}^{-1}$. The distribution of residual velocities can be easily explained in terms of a model of the Galaxy with a two-component outer ring $R_{1} R_{2}$ Mel'nik \& Rautiainen 2009; Rautiainen \& Mel'nik 2010; Mel'nik \& Rautiainen 2011). The residual velocities in other complexes are: $V_{R}=+6.9 \pm 2.7$ and $V_{T}=1.2 \pm 3.1$ (Local System), $V_{R}=-5.4 \pm 2.9$ and $V_{T}=+4.3 \pm 3.1$ (Carina) and $V_{R}=-4.6 \pm 1.5$ and $V_{T}=-11.5 \pm 1.4 \mathrm{~km} \mathrm{~s}^{-1}$ (Cygnus). The

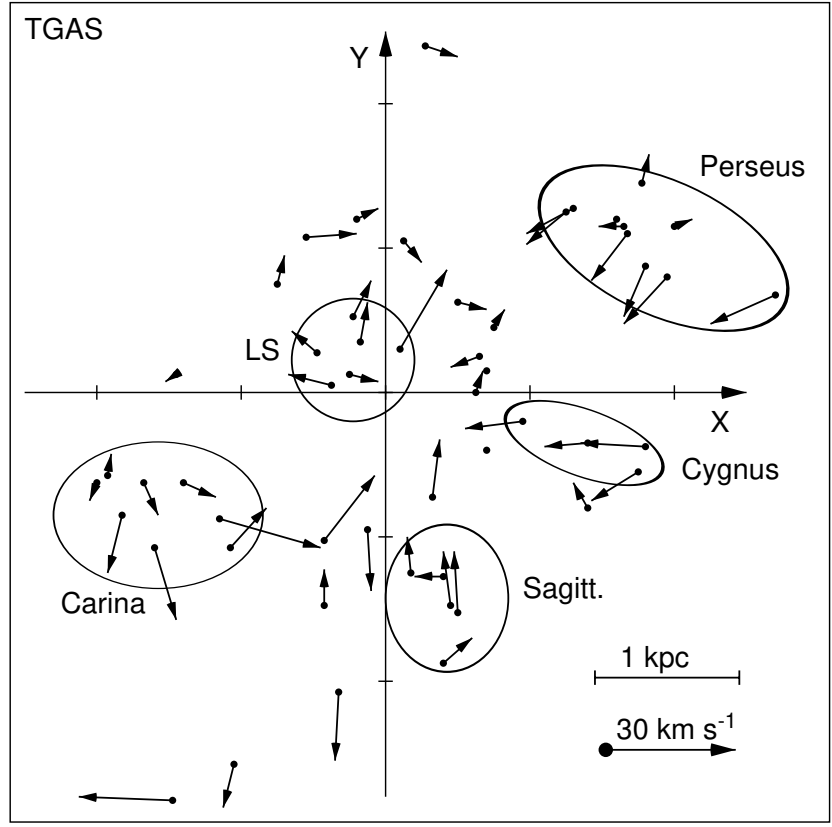

Figure 3. Distribution of the residual velocities of OBassociations in the Galactic plane. Shown are the residual velocities derived with kinematical data of at least two stars with known line-of-sight velocities and TGAS proper motions. OBassociations with residual velocities $\left|V_{R}\right|$ and $\left|V_{T}\right|$ smaller than 3 $\mathrm{km} \mathrm{s}^{-1}$ are shown as the black circles without any vector. The ellipses indicate the positions of the Sagittarius, Scorpio, Carina, Cygnus, Local System (LS), and Perseus stellar-gas complexes. The X-axis points in the direction of Galactic rotation and the $\mathrm{Y}$-axis is directed away from the Galactic centre. One tick interval along the $\mathrm{X}$ - and $\mathrm{Y}$-axis corresponds to $1 \mathrm{kpc}$. The Sun is at the origin.

rms difference of residual velocities in the stellar-gas complexes derived from TGAS and Hipparcos proper motions is less than $1 \mathrm{~km} \mathrm{~s}^{-1}$.

Currently, the accuracy of measured line-of-sight velocities of stars in OB-associations is less than the accuracy of sky-plane velocities derived with TGAS proper motions. The new epoch in the study of Galactic kinematics will begin when more accurate line-of-sight velocities are obtained for young stars by the Gaia radial-velocity spectrometer (Gaia Collaboration et al. 2016a). Of particular interest is the direction toward the Sagittarius arm (Grosbol 2016).

\subsection{Motions in the z-direction}

The residual velocities in the $\mathrm{z}$-direction are determined from the proper motions $\mu_{b}$ and line-of-sight velocities $V_{r}$ :

$V_{z}=4.74 \mu_{b} \cos b \cdot r+V_{r} \sin b+w_{0}$,

where $w_{0}$ is the velocity of the Sun along the Z-axis. Factor 4.74 transforms proper motions determined in units of mas $\mathrm{yr}^{-1}$ into $\mathrm{km} \mathrm{s}^{-1}$ provided $r$ is in $\mathrm{kpc}$.

We selected 53 OB-associations containing at least two stars with both known line-of-sight velocities and TGAS proper motions to determine their $V_{z}$ velocities and to calculate the most probable value of the solar velocity $w_{0}$, which appears to be $w_{0}=7.67 \pm 0.52 \mathrm{~km} \mathrm{~s}^{-1}$. The rms deviation 
Table 3. Parameters of the rotation curve

\begin{tabular}{|c|c|c|c|c|c|c|c|c|}
\hline Sample & $\begin{array}{c}\Omega_{0} \\
\mathrm{~km} \mathrm{~s}^{-1} \\
\mathrm{kpc}^{-1}\end{array}$ & $\begin{array}{c}\Omega_{0}^{\prime} \\
\mathrm{km} \mathrm{s}^{-1} \\
\mathrm{kpc}^{-2}\end{array}$ & $\begin{array}{c}\Omega_{0}^{\prime \prime} \\
\mathrm{km} \mathrm{s}^{-1} \\
\mathrm{kpc}^{-3}\end{array}$ & $\begin{array}{c}u_{0} \\
\mathrm{~km} \mathrm{~s}^{-1}\end{array}$ & $\begin{array}{c}v_{0} \\
\mathrm{~km} \mathrm{~s}^{-1}\end{array}$ & $\begin{array}{c}\mathrm{A} \\
\mathrm{km} \mathrm{s}^{-1} \\
\mathrm{kpc}^{-1}\end{array}$ & $\begin{array}{c}\sigma_{0} \\
\mathrm{~km} \mathrm{~s}^{-1}\end{array}$ & $N_{e q}$ \\
\hline $1\left(n_{v r} \geqslant 2, n_{\mu} \geqslant 2\right)$ & $\begin{array}{l}31.08 \\
\pm 0.86\end{array}$ & $\begin{array}{l}-4.75 \\
\pm 0.18\end{array}$ & $\begin{array}{c}1.11 \\
\pm 0.22\end{array}$ & $\begin{array}{c}7.27 \\
\pm 1.01\end{array}$ & $\begin{array}{l}10.63 \\
\pm 1.33\end{array}$ & $\begin{array}{l}17.83 \\
\pm 0.68\end{array}$ & 7.42 & 126 \\
\hline $2\left(n_{v r} \geqslant 5, n_{\mu} \geqslant 5\right)$ & $\begin{array}{l}31.31 \\
\pm 1.06\end{array}$ & $\begin{array}{l}-4.68 \\
\pm 0.22\end{array}$ & $\begin{array}{c}1.17 \\
\pm 0.31\end{array}$ & $\begin{array}{c}7.16 \\
\pm 1.28\end{array}$ & $\begin{array}{l}12.68 \\
\pm 1.77\end{array}$ & $\begin{array}{l}17.54 \\
\pm 0.86\end{array}$ & 7.69 & 82 \\
\hline
\end{tabular}

of velocities in the z-direction computed with TGAS catalog data is $\sigma_{v z}=3.80 \mathrm{~km} \mathrm{~s}^{-1}$, which is slightly less than the scatter of vertical velocity components computed using Hipparcos data, $\sigma_{v z}=5.0 \mathrm{~km} \mathrm{~s}^{-1}$.

Note that the $V_{z}$ velocities of OB-associations R 103 $(r=3.2 \mathrm{kpc})$ and Ara OB1B $(2.8 \mathrm{kpc})$ decrease in absolute value nearly twice: from $V_{z}=-31$ and $-24 \mathrm{~km} \mathrm{~s}^{-1}$ when computed with Hipparcos proper motions to $V_{z}=-15$ and $-8 \mathrm{~km} \mathrm{~s}^{-1}$ if determined using TGAS data. The other association with large $V_{z}$ velocity is Cyg OB8, $V_{z}=+11 \mathrm{~km}$ $\mathrm{s}^{-1}$. Excluding these three associations changes the value of $w_{0}$ to $7.45 \pm 0.38$ and decreases $\sigma_{v z}$ to $2.69 \mathrm{~km} \mathrm{~s}^{-1}$.

Interestingly, the $V_{z}$ velocity of OB-association Per OB1 is nearly zero $V_{z}=-0.9 \mathrm{~km} \mathrm{~s}^{-1}$ and though Per OB1 is located $\sim 100 \mathrm{pc}$ below the Galactic plane, $b=-3.15^{\circ}$, it does not move practically as a whole along the Z-axis.

\section{INTERNAL PROPERTIES OF OB-ASSOCIATIONS}

\subsection{Velocity dispersion in OB-associations with TGAS proper motions}

We compare the dispersions of proper motions of stars in OB-associations obtained with Hipparcos and TGAS data (Fig. 4). Here $\sigma_{\mu l}$ and $\sigma_{\mu b}$ are the robust estimates of the dispersions of proper motions in OB-associations derived in the following way. First, we sort the proper motions $\mu_{l}$ or $\mu_{b}$ of TGAS stars in OB-association to make rows of increasing values. Second, we exclude equal number of objects constituting the (1-0.68)/2 fraction of stars, $n_{\mu}$, from the upper and lower sides of the distribution. The remaining stars make up for nearly 68 percent of the number $n_{\mu}$. The half-width of the interval in the proper-motion components spanned by the remaining stars amounts to $\sigma_{\mu l}$ or $\sigma_{\mu b}$, respectively. It is an analog of the root-mean-squared velocity dispersion but it is less sensitive to outliers. We consider 18 OB-associations containing at least 10 stars with TGAS proper motions. When computed with TGAS data, the proper motion dispersions along l- and b-coordinates, $\sigma_{\mu l}$ and $\sigma_{\mu b}$, decrease significantly compared to the estimates obtained with Hipparcos proper motions: the reduction factor is $0.37 \pm 0.006$ and $0.40 \pm 0.006$ for 1 - and b-directions, respectively, or $0.39 \pm 0.04$ if averaged over both components. Note that $\sigma_{\mu l}$ of OB-association Cyg OB7 was excluded from

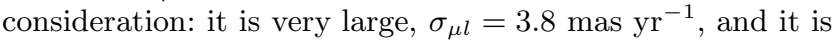
nearly the same when computed with TGAS and Hipparcos data. However, because of the small heliocentric distance

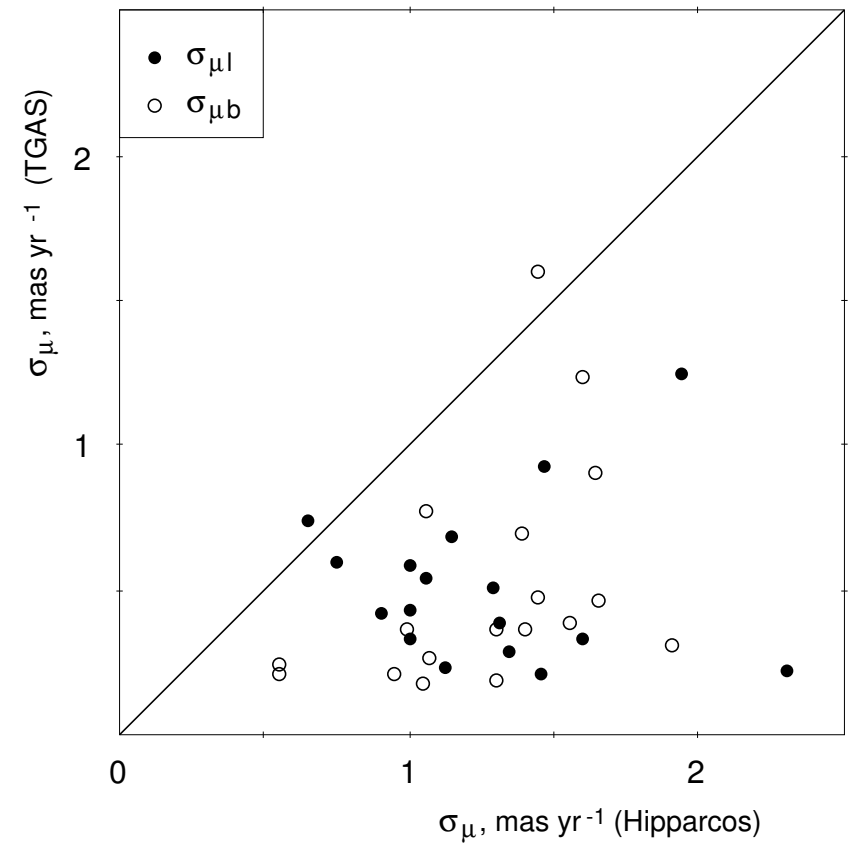

Figure 4. Comparison of the dispersions of proper motions inside OB-associations calculated with Hipparcos and TGAS data. Velocity dispersions along the 1- and b-coordinate, $\sigma_{\mu l}$ and $\sigma_{\mu b}$, are shown by the black and white circles, respectively. TGAS dispersions can be seen, on average, to be smaller than the corresponding Hipparcos dispersions. The $\sigma_{\mu l}=3.8 \mathrm{mas} \mathrm{yr}^{-1}$ of association Cyg OB7, nearly the same when computed with TGAS and Hipparcos proper motions, is excluded from consideration.

of Cyg OB7, $r=0.63 \mathrm{kpc}$, even this very large $\sigma_{\mu l}$ value translates into a velocity dispersion of only $\sigma_{v l}=11.4 \mathrm{~km}$ $\mathrm{s}^{-1}$.

We calculate the TGAS velocity dispersions, $\sigma_{v l}$ and $\sigma_{v b}$, along l- and b-coordinates as follows:

$\sigma_{v l}=4.74 r \sigma_{\mu l}$

$\sigma_{v b}=4.74 r \sigma_{\mu b}$,

Table 4lists the TGAS velocity dispersions, $\sigma_{v l}$ and $\sigma_{v b}$, obtained for $18 \mathrm{OB}$-associations containing at least 10 stars with TGAS proper motions, $n_{\mu} \geqslant 10$. It also provides the general information for OB-associations: the average Galactic coordinates, $l$ and $b$, heliocentric $(r)$ and Galactocentric distance $(R)$ distances.

The average TGAS velocity dispersions in OBassociations are $\overline{\sigma_{l}}=4.3$ and $\overline{\sigma_{b}}=3.4 \mathrm{~km} \mathrm{~s}^{-1}$. It is cur- 


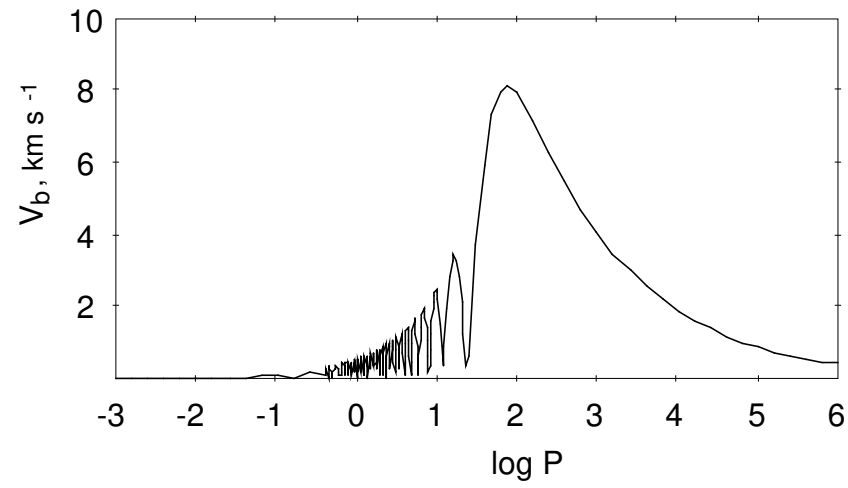

Figure 5. Dependence of the additional velocity $V_{b}$ on $\log P$. Binary system considered includes two stars of mass $10 \mathrm{M}_{\odot}$ rotating on a circular orbit around their common mass center with period $P$. The velocity $V_{b}$ is the maximal contribution of a binary system into the TGAS velocity dispersion.

rently not clear why the velocity dispersions in Cyg OB8 $\left(\sigma_{b}=13.9\right)$ and in Cyg OB7 $\left(\sigma_{l}=11.4\right)$ are so high.

Generally, the Cygnus region is difficult to analyze because of the high concentration of bright stars in the sky plane. The large velocity dispersions in associations Cyg OB7 $\left(\sigma_{v l}\right)$ and Cyg OB8 $\left(\sigma_{v b}\right)$ compared to other associations can also be seen in Figure 6

The fact that the TGAS velocity dispersions inside OB-associations drops to $4 \mathrm{~km} \mathrm{~s}^{-1}$, whereas the standard deviation, $\sigma_{0}$, of the velocities of OB-associations from the rotation curve remains at the level $7-8 \mathrm{~km}$ $\mathrm{s}^{-1}$ (Table 3) indicates that OB-associations identified by Blaha \& Humphreys (1989) mainly include stars born from the same molecular cloud.

Due to the turbulent motions inside giant molecular clouds, the velocity dispersion inside a cloud must grow with the size $S$ of the region considered as $\sigma_{v} \sim S^{p}$. The study by Solomon et al. (1987) suggests that power index $p$ is close to $p \approx 0.5$. If we suppose that the velocity dispersion in a giant cloud with a diameter of $10 \mathrm{pc}$ (the average size of open clusters) is $1-2 \mathrm{~km} \mathrm{~s}^{-1}$, then it must be $\sim 3$ times larger for clouds of $100 \mathrm{pc}$ in diameter (the average size of OB-associations), i.e. $3-6 \mathrm{~km} \mathrm{~s}^{-1}$. So the value of $\sigma_{v}=4$ $\mathrm{km} \mathrm{s}^{-1}$ is consistent with the properties of giant molecular clouds.

\subsection{Binary stars}

Mason et al. (1998) find that the majority (> 59 percent) of $\mathrm{O}$ stars in clusters and OB-associations are binary. In principle, binary stars can inflate the observed velocity dispersion inside OB-associations derived with TGAS proper motions, which are calculated from the positions obtained with the time difference $T=24 \mathrm{yr}$. The main contribution into TGAS proper motions must be provided by binary systems with orbital periods of $P \approx 50 \mathrm{yr}$, which corresponds to the component of the binary system moving to the opposite points of their orbit between the epochs of Hipparcos and Gaia.

Let us calculate the upper limit for the probable contribution of binary stars, $\sigma_{b}$, into the observed dispersion of TGAS proper motions inside OB-associations. For simplicity we consider a binary system consisting of two components of mass $M_{b}=10 \mathrm{M}_{\odot}$ moving in circular orbits around their common mass center with period $P$. A $10 \mathrm{M}_{\odot}$-star can be considered as a representative of stars in OB-associations from the list by Blaha \& Humphrevs (1989): most of stars listed in the catalog (about 2/3) have spectral types in the B0-B2 interval corresponding to the mass range $8-15 M_{\odot}$ (Hohle, Neuhäuser \& Schutz 2010).

The distance between the component stars $D$ can be estimated from Newton's gravity law:

$\frac{M_{b} \cdot V_{o r b}^{2}}{D / 2}=\frac{G \cdot M_{b}^{2}}{D^{2}}$,

or, given that

$V_{\text {orb }}=\pi \cdot D / P$,

we have

$\frac{D^{3}}{P^{2}}=\frac{G \cdot M_{b}}{2 \pi^{2}}$,

or

$D=\left(\frac{G}{2 \pi^{2}}\right)^{1 / 3} \cdot P^{2 / 3} \cdot M_{b}^{1 / 3}$,

The distance between components $D$ is the maximal possible shift of the star between the two epochs of observations. However, the real displacement is smaller because the star positions at the two epochs are practically always less than the orbit diameter apart. The length of the chord connecting the initial and final position of the star in its circular orbit is (by law of cosines):

$S=D \cdot \sqrt{\frac{\left(1-\cos \left(2 \pi \frac{T}{P}\right)\right)}{2}}$.

The maximum possible extra velocity along any given direction due to the orbital displacement of this component is equal to

$V_{b}=\frac{S}{T}$,

or, in view of eqs. (10) and (11):

$V_{b}=6.0 \cdot P^{2 / 3} \cdot M_{b}^{1 / 3} T^{-1} \cdot \sqrt{\frac{\left(1-\cos \left(2 \pi \frac{T}{P}\right)\right)}{2}}$

where $V_{b}$ is in $\mathrm{km} \mathrm{s}^{-1}, M_{b}$ is in solar masses, $P$ and $T$ are in years.

Figure 5 shows the dependence of the maximum additional velocity $V_{b}$ on $\log P$ for $M_{b}=10 M_{\odot}$ and $T=24 \mathrm{yr}$. The velocity $V_{b}$ reaches maximum of $8.1 \mathrm{~km} \mathrm{~s}^{-1}$ at period $\sim 80 \mathrm{yr}$, because the radius of the orbit increases with increasing $P$. The decrease of $V_{b}$ at larger $P$ is caused by the fact that a star can pass only a small part of its orbit for 24-year time interval. On the other hand, the decrease of $V_{b}$ at smaller $P$ is due to decreasing distance $D$ between binary stars, $D \sim P^{2 / 3}$. The velocity $V_{b}$ demonstrates oscillations with zero values corresponding to the periods $P=T / n$, where $n$ is integer and the binary star makes a whole number of revolutions in time interval $T$, resulting in zero displacement $D$.

To estimate the effective contribution of binary stars into the velocity dispersion inside $\mathrm{OB}$-association, $\sigma_{v}$, we should consider the fraction of multiple stars, $f_{b}$, in OBassociations, the factor measuring the average projection, 
$f_{j}$, of the shift $S$ into l- or b-direction, distribution of binary periods $f_{p}(\log P)$, and the additional velocity due to orbital motion, $V_{b}^{2}(\log P)$, as a function of $\log P$.

Sana (2017) overviews recent estimates of the fraction of binary stars among OB-stars, which vary from $f_{b}=0.2$ to 0.7 . We adopt $f_{b}=0.5$ as some average value.

If we suppose that the ensemble the binary systems in an OB-association is oriented randomly with respect to the line of sight then the effective value of the squared projection of the orbital displacement $S_{l}^{2}$ or $S_{b}^{2}$ onto the longitude or latitude direction, respectively, is equal to $S^{2} / 3$, hence $f_{j}=1 / 3$.

Aldoretta et al. (2015) show that the period distribution of massive binary stars is approximately flat in increments of $\log P$ (Öpik's law) in the interval of $\log P$ from -3 to +6 . So we can suppose that $f_{p}=1 / 9$ for unit of $\log P$.

Generally, we can write:

$\sigma_{b}^{2}=f_{b} \cdot f_{j} \cdot \int_{\log P=-3}^{\log P=+6} V_{b}^{2}(\log P) \cdot f_{p}(\log P) \cdot d \log P$.

We now use the values of $f_{b}=0.5, f_{j}=1 / 3, f_{p}=1 / 9$ and the distribution of $V_{b}$ over $\log P$ (Fig. 5 ) to calculate the effective contribution of binary stars into the TGAS velocity dispersion (Eq. 14), which appears to be $\sigma_{b}=1.19 \mathrm{~km} \mathrm{~s}^{-1}$, or

$\sigma_{b}=1.19 \cdot\left(M_{b} / 10 M_{\odot}\right)^{1 / 3}$

for a binary component of mass $M_{b}$. This value is rather small compared to $\sigma_{v}=4 \mathrm{~km} \mathrm{~s}^{-1}$ caused by turbulent motions inside giant molecular clouds.

\subsection{Virial and stellar masses of OB-associations}

Giant molecular clouds are supposed to be close to their virial equilibrium (Larson 1981; Krumholz. Matzner \& McKee 2006) and therefore the velocity dispersions of stars in OB-associations must correspond to the masses of their parent giant molecular clouds. The high precision of TGAS proper motions allows estimating the virial masses of OB-associations. The virial condition between the kinetic $\mathrm{K}$ and potential $\mathrm{U}$ energies of a system is:

$2 K+U=0$.

The potential energy of a uniform sphere of radius $a$ and constant density $\rho$ is :

$U=-\int_{0}^{a} \frac{\left(\frac{4}{3} G \pi \rho r^{3}\right)\left(4 \pi \rho r^{2} d r\right)}{r}=-\frac{3 G M^{2}}{5 a}$,

where $\mathrm{M}$ is the mass of the sphere:

$M=\frac{4 \pi a^{3} \rho}{3}$.

The kinetic energy of a system with one-dimensional velocity dispersion $\sigma_{v}$ is :

$K=\frac{3 M \sigma_{v}^{2}}{2}$.

Hence, the virial mass of the parent giant molecular cloud can be calculated in the following way:
$M_{v i r}=\frac{5 a \sigma_{v}^{2}}{G}$,

where $\sigma_{v}$ and $a$ are determined from observations.

There is no problem with observed one-dimensional velocity $\sigma_{v}$, it can be estimated as the average of $\sigma_{v l}$ and $\sigma_{v b}$ :

$\sigma_{v}=\left(\sigma_{v l}+\sigma_{v b}\right) / 2$.

However, the so-called radius $a$ of a parent molecular cloud can be treated in different ways. We calculate $a$ as the radius containing 68 percent of the association member stars. Here we consider all members of OB-associations with known photometric measurements and not just TGAS stars. We also suppose that the sizes of OB-associations have not changed significantly since the epoch of star formation. However, some OB-associations appear to be expanding, so in these cases, the radius $a$ needs a correction (for more details see Section 4.3).

Table 4 also gives values of $M_{v i r}$ and $a$. The median value of $M_{v i r}$ for 18 OB-associations considered is $7.110^{5}$ $\mathrm{M}_{\odot}$ which is consistent with the masses of giant molecular clouds $10^{5}-10^{6} \mathrm{M}_{\odot}($ Sanders, Scoville \& Solomon 1985).

Note that in our calculations of virial masses we used the values of $\sigma_{v}$ corrected for the effect of binary stars:

$\sigma_{\text {cor }}^{2}=\sigma_{v}^{2}-\sigma_{b}^{2}$.

To estimate the stellar masses of OB-associations we use the multi-component power-law distribution derived by Kroupa (2002). The number of stars $d N(M)$ in the mass range $\Delta M$ is determined for three mass domains by the following laws:

$$
d N(M)=C_{0} \quad \begin{cases}C_{1} \cdot M^{-0.3} & 0.01<M / M_{\odot}<0.08 \\ C_{2} \cdot M^{-1.3} & 0.08<M / M_{\odot}<0.5 \\ C_{3} \cdot M^{-2.3} & 0.5<M / M_{\odot}<\infty .\end{cases}
$$

The coefficients $C_{1}, C_{2}$, and $C_{3}$ are calculated from the continuity condition at the boundaries of the mass domains: $C_{1}=0.469, C_{2}=0.038$ and $C_{3}=0.019$.

We also assume that the catalog by Blaha \& Humphrevs (1989) includes all stars of OBassociations with masses greater than $20 M_{\odot}$ or it is essentially complete down to the absolute magnitude $M_{V}<-4.0^{m}$. To determine the mass of stars in OBassociations we use the mass-absolute magnitude relation by Bressan et al. (2012). This relation is monotonic in the range $-7.8<M_{V}<+0.50$ and corresponds to the mass range $3-50 \mathrm{M}_{\odot}$, which covers 99.3 percent of OB-association stars listed in the catalog by Blaha \& Humphrevs (1989). The age of the brightest OB-stars is supposed to be $4 \mathrm{Myr}$.

The value of $C_{0}$ is derived from the following calibration:

$N_{20}=C_{0} C_{3}\left(20^{-1.3}-50^{-1.3}\right) / 1.3$,

where $N_{20}$ is the number of stars with masses $M>20 \mathrm{M}_{\odot}$ in a particular OB-association.

Table 4 also gives the stellar masses $M_{s t}$ of OBassociations and the number $N_{20}$ of stars with masses $M>$ $20 \mathrm{M}_{\odot}$. The median value of $M_{s t}$ calculated for $18 \mathrm{OB}$ associations is $9.010^{3} \mathrm{M}_{\odot}$. Note that in the case of pure Salpiter (1955) distribution, $d N \sim M^{-2.35} d M$, in the mass interval from 0.08 (Grossman \& Graboske 1971) to $50 \mathrm{M}_{\odot}$ we obtain nearly twice greater $M_{s t}$ values with the median of $1910^{3} \mathrm{M}_{\odot}$. 
For each OB-association from Table 4 we can estimate the average efficiency of star formation in its parent molecular cloud, which is equal to the ratio of the stellar mass to the gaseous mass of the giant cloud:

$\epsilon=M_{s t} / M_{v i r}$.

Table 4 shows that $\epsilon$ values vary from 0.1 to 13.6 percent with the median value of 2.1 percent which agrees with other estimates (Myers et al. 1986; Evans et al. 2009; Garcia et al. 2014).

Note that the estimates of star-formation efficiency $\epsilon$ obtained here refer to the entire volume of OB-associations, and $\epsilon$ can be smaller or larger in different parts of their parent giant molecular cloud.

\subsection{Present-day mass of OB-associations and their unboundness}

The fact that virial mass exceeds the stellar mass by more than 70 times supports the conclusion that OB-associations are unbound objects. Some doubts concern the gas which OB-associations could harbor inside their volumes, so the real masses of OB-associations can be conspicuously larger than those of the stellar component.

Numerical simulations demonstrate that stars with masses $M \geqslant 20 \mathrm{M}_{\odot}$ destroy crucially molecular clouds of masses up to a few times $10^{4}$ $\mathrm{M}_{\odot} \quad$ Colin, Vazquez-Semadeni \& Gomez 2013; Dale, Ercolano \& Bonnell 2012).

Table 4 shows that of the 18 OB-associations considered nine contain more than 20 stars with masses $M \geqslant 20 \mathrm{M}_{\odot}$. On the other hand, masses of their parent molecular clouds are $10^{5}-10^{6} \mathrm{M}_{\odot}$, implying the average escape velocity of 12 $\mathrm{km} \mathrm{s}^{-1}$, and hence the full evaporation of gas clouds seems to be impossible.

Dale, Ercolano \& Bonnell (2012) simulate the starformation processes in the molecular cloud with mass $10^{6}$ $\mathrm{M}_{\odot}$ and initial radius of $180 \mathrm{pc}$. They found that after 3 Myr of ionization the surface density $\Sigma$ of the cloud is $\sim 2$ $10^{-3} \mathrm{~g} \mathrm{~cm}^{-2}$, which corresponds to the volume density of $\sim 1$ atom $\mathrm{cm}^{-3}$.

Cappa \& Herbstmeier (2000) study the distribution of HI gas in the vicinity of association Per OB1. Their Figure 2 indicates that HI surface density inside Per OB1 is $\Sigma \sim 3$ $10^{20}$ atoms $\mathrm{cm}^{-2}$. If the size of Per OB1 along the line of sight is $\sim 200$ pc then the HI volume density must be $\sim 0.5$ atom $\mathrm{cm}^{-3}$.

Let us suppose that the fraction of molecular gas inside the volume of OB-associations is negligible, but neutral hydrogen with the volume density $\rho_{H}$ equal to 1 atom $\mathrm{cm}^{-3}$ can be present there. Then the upper estimate for the present-day mass of OB-association, $M_{t}$, includes the mass of the stellar component, $M_{s t}$, and the mass of HI possibly located inside the volume of the OB-association:

$M_{t}=M_{s t}+M_{g}$.

The gas mass $M_{g}$ can be estimated from the following formula:

$M_{g}=\frac{4 \pi}{3} a^{3} \rho_{H}$

Table 4 also lists the masses of the gaseous component,
$M_{g}$. The median gas mass appears to be $18.310^{3} \mathrm{M}_{\odot}$, and hence the contribution of gas to the total mass is usually comparable to the mass of the stellar component. The exception is two extremely extended OB-associations: Cep OB1 and NGC 2439, where $M_{g}$ exceeds $M_{s t}$ by a factor of more than 30, however, even in these cases the total masses of OB-associations, $M_{t}$, are nearly 10 times smaller than the corresponding virial masses, $M_{v i r}$.

We can thus conclude that OB-associations must be unbound objects provided they do not contain a lot of dense gas.

\subsection{Tidal radius of OB-associations}

Given the known present-day masses of OB-associations, we can estimate their tidal radii, $r_{t d}$, at which the gravitational force produced by the OB-association equals the tidal perturbation from the Galaxy.

Let us consider the forces applied to a test particle located between the OB-association and the Galactic centre. Subscripts 1 and 2 refer to the distances and velocities of the test particle and the centre of the OB-association, respectively. The distance between the test particle and the centre of the OB-association is $\Delta R=R_{1}-R_{2}<0$. The test particle is subject to two forces working in the opposite directions: the gravity from the Galaxy, $\Omega_{1}^{2} R_{1}$, and the gravity from the OB-association, $G M /(\Delta R)^{2}$. If the test particle is bound with the OB-association, then it rotates around the Galactic centre at the angular velocity of the OB-association, $\Omega_{2}$. Here we ignore the velocity of the test particle due to the gravity of the OB-association. The acceleration of the test particle is equal to the sum of its centripetal acceleration, $\Omega_{2}^{2} R_{1}$, and additional acceleration determined with respect to the centre of the OB-association, $\ddot{R}_{1}$. Newton's law gives us the following equation:

$\ddot{R}_{1}-\Omega_{2}^{2} R_{1}=\frac{G M}{(\Delta R)^{2}}-\Omega_{1}^{2} R_{1}$,

where $M$ is the mass of the OB-association, $M_{t}$. The tidal radius, $r_{t d}$ is derived from the condition $\ddot{R}_{1}=0$. By assuming $\Delta R / R_{2} \ll 1$, we obtain the following estimate:

$r_{t d}=|\Delta R|=\left(\frac{G M}{4 A \Omega}\right)^{1 / 3}$

where $A$ is Oort's constant $A=-0.5 R \Omega^{\prime}(R)$ at the Galactocentric distance $R$.

The formula for the tidal force, $T=-4 A \Omega \Delta R$, can also be derived from basic equations by Goldreich \& Tremaine (1980, Eq. 32), it is also discussed in Stark \& Blitz (1978, Eq. 1).

We calculate the tidal radii $r_{t d}$ for 18 OB-associations containing at least 10 stars with TGAS proper motions. The angular velocity $\Omega$ and its first derivative $\Omega^{\prime}$ at the Galactocentric radius $R$ of each OB-association are computed based on the parameters of the rotation curve given in Table 3. The median tidal radius $r_{t d}$ appears to be $40 \mathrm{pc}$, which is a bit smaller than the median radius $a$ of OB-associations, 56 pc. On average, 27 percent of stars of OB-associations must lie outside their tidal radii and tidal perturbations from the Galaxy are essential for them.

We also calculate the minimal value of the tidal radius 
Kinematics of OB-associations

Table 4. Virial and stellar masses of OB-associations, $M_{v i r}$ and $M_{s t}$, star formation efficiency $\epsilon$

\begin{tabular}{|c|c|c|c|c|c|c|c|c|c|c|c|c|c|c|}
\hline Name & $\begin{array}{r}l \\
\text { deg. }\end{array}$ & $\begin{array}{r}b \\
\text { deg. }\end{array}$ & $\begin{array}{c}r \\
\mathrm{kpc}\end{array}$ & $\begin{array}{c}R \\
\mathrm{kpc}\end{array}$ & $\begin{array}{c}\sigma_{v l} \\
\mathrm{~km} \mathrm{~s}^{-1}\end{array}$ & $\begin{array}{c}\sigma_{v b} \\
\mathrm{~km} \mathrm{~s}^{-1}\end{array}$ & $\begin{array}{c}a \\
\mathrm{pc}\end{array}$ & $\begin{array}{r}r_{t d} \\
\mathrm{pc}\end{array}$ & $\begin{array}{r}M_{v i r} \\
M_{\odot}\end{array}$ & $n_{\mu}$ & $\begin{array}{l}M_{s t} \\
M_{\odot}\end{array}$ & $N_{20}$ & $\begin{array}{c}M_{g} \\
M_{\odot}\end{array}$ & $\epsilon 100 \%$ \\
\hline SGR OB1 & 7.55 & -0.77 & 1.26 & 6.25 & 1.3 & 4.6 & 42 & 24 & $3.610^{5}$ & 13 & $9.610^{3}$ & 23 & $7.610^{3}$ & 2.7 \\
\hline CYG OB1 & 75.84 & 1.12 & 1.46 & 7.28 & 3.8 & 2.5 & 32 & 31 & $3.110^{5}$ & 12 & $16.710^{3}$ & 40 & $3.510^{3}$ & 5.3 \\
\hline CYG OB8 & 77.91 & 3.36 & 1.83 & 7.34 & 3.7 & 13.9 & 42 & 24 & $36.810^{5}$ & 10 & $7.110^{3}$ & 17 & $7.410^{3}$ & 0.2 \\
\hline CYG OB7 & 88.99 & 0.03 & 0.63 & 7.52 & 11.3 & 2.0 & 53 & 19 & $26.410^{5}$ & 16 & $3.310^{3}$ & 8 & $15.610^{3}$ & 0.1 \\
\hline CEP OB3 & 110.42 & 2.56 & 0.70 & 7.77 & 1.7 & 1.4 & 12 & 14 & $0.110^{5}$ & 13 & $1.210^{3}$ & 3 & $0.210^{3}$ & 10.0 \\
\hline PER OB1 & 134.67 & -3.15 & 1.83 & 8.88 & 5.0 & 3.1 & 59 & 48 & $8.210^{5}$ & 58 & $36.210^{3}$ & 87 & $20.910^{3}$ & $4.4^{*}$ \\
\hline CAS OB6 & 135.02 & 0.75 & 1.75 & 8.83 & 2.4 & 3.9 & 78 & 29 & $7.810^{5}$ & 11 & $7.910^{3}$ & 19 & $49.410^{3}$ & 1.0 \\
\hline CAM OB1 & 141.08 & 0.89 & 0.80 & 8.14 & 4.7 & 3.4 & 86 & 23 & $15.310^{5}$ & 26 & $5.010^{3}$ & 12 & $66.010^{3}$ & 0.3 \\
\hline AUR OB1 & 173.83 & 0.14 & 1.06 & 8.55 & 2.6 & 1.5 & 69 & 22 & $2.210^{5}$ & 12 & $3.710^{3}$ & 9 & $33.910^{3}$ & 1.7 \\
\hline MON OB2 & 207.46 & -1.65 & 1.21 & 8.59 & 1.3 & 2.6 & 71 & 25 & $2.010^{5}$ & 10 & $5.810^{3}$ & 14 & $36.610^{3}$ & 2.9 \\
\hline CEN OB1 & 304.14 & 1.44 & 1.92 & 6.62 & 5.2 & 1.9 & 69 & 32 & $9.110^{5}$ & 28 & $21.210^{3}$ & 51 & $34.410^{3}$ & 2.3 \\
\hline
\end{tabular}

* Values of $M_{v i r}$ and $\epsilon$ for Per OB1 and Car OB1 are corrected for the expansion effect (Section 4.3)

Table 5. Expansion/compression of OB-associations with TGAS proper motions

\begin{tabular}{|c|c|c|c|c|c|c|c|c|c|}
\hline Name & $\begin{array}{c}p_{l} \\
\mathrm{~km} \mathrm{~s}^{-1} \mathrm{kpc}^{-1}\end{array}$ & $\begin{array}{c}p_{b} \\
\mathrm{~km} \mathrm{~s}^{-1} \mathrm{kpc}^{-1}\end{array}$ & $\begin{array}{c}a \\
\mathrm{pc}\end{array}$ & $\begin{array}{c}e_{1} \\
\mathrm{~km} \mathrm{~s}^{-1}\end{array}$ & $\begin{array}{c}u_{l} \\
\mathrm{~km} \mathrm{~s}^{-1}\end{array}$ & $\begin{array}{c}u_{b} \\
\mathrm{~km} \mathrm{~s}^{-1}\end{array}$ & $\begin{array}{c}\tilde{u}_{l} \\
\mathrm{~km} \mathrm{~s}^{-1}\end{array}$ & $\begin{array}{c}\tilde{u}_{b} \\
\mathrm{~km} \mathrm{~s}^{-1}\end{array}$ & $n_{\mu}$ \\
\hline SGR OB1 & $70 \pm 38$ & $238 \pm 26$ & 42 & $0.3 \pm 0.1$ & $2.9 \pm 1.6$ & $10.0 \pm 1.1$ & $2.6 \pm 1.6$ & $9.7 \pm 1.1$ & 13 \\
\hline CYG OB3 & $139 \pm 138$ & $159 \pm 116$ & 26 & $0.1 \pm 0.0$ & $3.6 \pm 3.6$ & $4.1 \pm 3.0$ & $3.5 \pm 3.6$ & $\overline{4.0 \pm 3.0}$ & 15 \\
\hline CYG OB1 & $-57 \pm 102$ & $112 \pm 131$ & 32 & $0.3 \pm 0.1$ & $-1.8 \pm 3.3$ & $3.6 \pm 4.2$ & $-2.1 \pm 3.3$ & $3.3 \pm 4.2$ & 12 \\
\hline CYG OB8 & $-45 \pm 55$ & $186 \pm 152$ & 42 & $0.5 \pm 0.1$ & $-1.9 \pm 2.3$ & $7.7 \pm 6.3$ & $-2.4 \pm 2.3$ & $7.3 \pm 6.3$ & 10 \\
\hline CYG OB7 & $-39 \pm 85$ & $63 \pm 26$ & 53 & $0.8 \pm 0.2$ & $-2.1 \pm 4.5$ & $3.4 \pm 1.4$ & $-2.9 \pm 4.5$ & $2.6 \pm 1.4$ & 16 \\
\hline CEP OB2 & $77 \pm 26$ & $11 \pm 26$ & 45 & $1.1 \pm 0.1$ & $3.5 \pm 1.2$ & $0.5 \pm 1.2$ & $2.4 \pm 1.2$ & $-0.6 \pm 1.2$ & 34 \\
\hline CEP OB1 & $56 \pm 19$ & $1 \pm 39$ & 178 & $3.7 \pm 0.1$ & $9.9 \pm 3.4$ & $0.1 \pm 6.9$ & $6.2 \pm 3.4$ & $-3.6 \pm 6.9$ & 20 \\
\hline CEP OB3 & $-39 \pm 48$ & $71 \pm 67$ & 12 & $0.4 \pm 0.0$ & $-0.5 \pm 0.6$ & $0.9 \pm 0.8$ & $-0.9 \pm 0.6$ & $0.5 \pm 0.8$ & 12 \\
\hline PER OB1 & $45 \pm 15$ & $103 \pm 22$ & 59 & $1.4 \pm 0.0$ & $2.7 \pm 0.9$ & $6.1 \pm 1.3$ & $1.3 \pm 0.9$ & $\underline{4.7 \pm 1.3}$ & 58 \\
\hline CAS OB6 & $9 \pm 47$ & $227 \pm 80$ & 78 & $1.9 \pm 0.1$ & $0.7 \pm 3.7$ & $17.8 \pm 6.3$ & $-1.2 \pm 3.7$ & $1 \overline{5.9 \pm 6.3}$ & 11 \\
\hline CAM OB1 & $34 \pm 20$ & $3 \pm 28$ & 86 & $1.2 \pm 0.2$ & $2.9 \pm 1.8$ & $0.3 \pm 2.4$ & $1.7 \pm 1.8$ & $-0.9 \pm 2.4$ & 26 \\
\hline AUR OB1 & $-36 \pm 27$ & $19 \pm 10$ & 69 & $0.1 \pm 0.3$ & $-2.5 \pm 1.9$ & $1.3 \pm 0.7$ & $-2.6 \pm 1.9$ & $1.2 \pm 0.7$ & 12 \\
\hline MON OB2 & $-278 \pm 77$ & $232 \pm 203$ & 71 & $-1.3 \pm 0.2$ & $-19.7 \pm 5.4$ & $16.5 \pm 14.4$ & $-18.4 \pm 5.4$ & $17.8 \pm 14.4$ & 10 \\
\hline NGC 2439 & $64 \pm 42$ & $-106 \pm 21$ & 155 & $-2.8 \pm 0.0$ & $10.0 \pm 6.6$ & $-16.4 \pm 3.2$ & $\overline{12.8 \pm 6.6}$ & $-13.6 \pm 3.2$ & 10 \\
\hline CAR OB1 & $118 \pm 43$ & $111 \pm 28$ & 63 & $0.2 \pm 0.1$ & $7.5 \pm 2.7$ & $7.0 \pm 1.8$ & $7.3 \pm 2.7$ & $6.8 \pm 1.8$ & 15 \\
\hline CAR OB2 & $-100 \pm 136$ & $42 \pm 64$ & 28 & $0.1 \pm 0.0$ & $-2.8 \pm 3.8$ & $1.2 \pm 1.8$ & $-\overline{2.9 \pm 3.8}$ & $\overline{1.1 \pm 1.8}$ & 10 \\
\hline CRU OB1 & $-29 \pm 47$ & $29 \pm 23$ & 40 & $0.1 \pm 0.0$ & $-1.2 \pm 1.9$ & $1.2 \pm 0.9$ & $-1.3 \pm 1.9$ & $1.1 \pm 0.9$ & 17 \\
\hline CEN OB1 & $-87 \pm 33$ & $35 \pm 18$ & 69 & $0.7 \pm 0.1$ & $-6.0 \pm 2.3$ & $2.4 \pm 1.3$ & $-6.7 \pm 2.3$ & $1.7 \pm 1.3$ & 27 \\
\hline
\end{tabular}

(Table 4) assuming that the mass of the OB-association consists of stellar component exclusively, $M_{t}=M_{s t}$. The tidal radii of OB-associations then drop to the values in the $14-$ $36 \mathrm{pc}$ interval with the median of $26 \mathrm{pc}$. It means that 39 percent of member stars of OB-associations must be located outside their tidal radii.

Thus, two different estimates of tidal radii of OBassociations yield nearly the same result: $\sim 1 / 3$ of stars of OB-associations must lie outside their tidal radius.

\section{EXPANSION OF OB-ASSOCIATIONS}

\subsection{Determination of the parameters of expansion/compression of OB-associations}

We use TGAS proper motions to study possible expansion/compression of OB-associations in the l- and bdirection. The parameters of expansion/compression $p_{l}$ and $p_{b}$ are calculated from the following equations:

$4.74 \mu_{l} r=v_{l 0}+p_{l} \cdot r \sin \left(l-l_{0}\right)$,

$4.74 \mu_{b} r=v_{b 0}+p_{b} \cdot r \sin \left(b-b_{0}\right)$, 
where $v_{l 0}$ and $v_{b 0}$ are the average velocities of the association; $l_{0}$ and $b_{0}$ are the coordinates of the centre of the association, and parameters $p_{l}$ or $p_{b}$ characterize expansion/compression along the l- or b-direction (positive and negative values correspond to expansion and compression, respectively).

The observed specific velocities of expansion or compression, $u_{l}$ and $u_{b}$, are calculated in following way:

$u_{l}=p_{l} \cdot a$,

$u_{b}=p_{b} \cdot a$.

Note that expansion/compression of OB-associations requires some caution in interpretation: the motion of an association as a whole with the line-of-sight velocity $V_{r}$ (see Table 1) can cause the effect of spurious expansion/compression. The velocity of spurious expansion/compression, $e_{1}$, caused by the line-of-sight velocity $V_{r}$ is determined by the following expression:

$e_{1}=-V_{r} \cdot \frac{a}{r}$,

where the negative line-of-sight velocities $V_{r}$ produces spurious expansion, $e_{1}>0$, while positive $V_{r}$ gives spurious compression, $e_{1}<0$.

Table 5 lists the parameters of observed expansion/compression, $p_{l}$ and $p_{b}$, the radius of OB-associations, $a$, the velocity of spurious expansion/compression $e_{1}$, the observed specific velocities of expansion/compression, $u_{l}$ and $u_{b}$, and the number of stars with known TGAS proper motions, $n_{\mu}$. We can see that the velocity of spurious expansion/compression, $e_{1}$, can reach $\sim 4 \mathrm{~km} \mathrm{~s}^{-1}$ (Cep OB1). So the observed velocities of expansion/compression, $u_{l}$ or $u_{b}$, should be corrected for this effect:

$\tilde{u}_{l}=u_{l}-e 1$,

$\tilde{u}_{b}=u_{b}-e 1$.

The corrected velocities of expansion/compression, $\tilde{u}_{l}$ and $\tilde{u}_{b}$, are listed in Table 5 as well. Further, we will discuss only velocities, $\tilde{u}_{l}$ and $\tilde{u}_{b}$, determined at $P>2.5 \sigma$ confidence level, which are underlined in Table 5

Figures [6] and 7 show the distribution of the relative velocities of stars inside OB-association, $v_{l}^{\prime}$ and $v_{b}^{\prime}$, calculated with respect to its average velocities $v_{l 0}$ and $v_{b 0}$ :

$v_{l}^{\prime}=4.74 r \mu_{l}-v_{l 0}$

$v_{b}^{\prime}=4.74 r \mu_{b}-v_{b 0}$

Note that we excluded from consideration stars whose relative velocities exceed $50 \mathrm{~km} \mathrm{~s}^{-1}$, i. e. $\left|v_{l}^{\prime}\right|>50$ or $\left|v_{b}^{\prime}\right|>50 \mathrm{~km} \mathrm{~s}^{-1}: \mathrm{BD}+57$ 530A (Per OB1), HD 212043 (Cep OB2), HD 192445 (Cyg OB3), HD 216878 (Cep OB3), $\mathrm{BD}+233843$ (Vul OB1), HDE 328209 (Ara OB1B), HD 172488 (Sct OB2), and HD 112272 (Cen OB1). Stars BD +57 530A (Per OB1) and HD 212043 (Cep OB2) have parallaxes $\pi=12.44 \pm 0.28$ and $4.91 \pm 0.63$, which correspond to distances $r=0.1$ and $0.2 \mathrm{kpc}$, so they cannot belong to the associations Per OB1 and Cep OB2 located at distances $r=1.8$ and $0.7 \mathrm{kpc}$, respectively. The parallaxes of other aforementioned stars are consistent with their membership in the corresponding associations. Generally, they can be binary or runaway stars which can form quite frequently in young clusters (for example, Fuiii \& Portegies Zwart 2011).
Table 6. Kinematic ages of OB-associations

\begin{tabular}{lcccc}
\hline Name & $\begin{array}{c}T_{l} \\
\text { Myr }\end{array}$ & $\begin{array}{c}T_{b} \\
\text { Myr }\end{array}$ & $\begin{array}{c}T_{l}^{*} \\
\text { Myr }\end{array}$ & $\begin{array}{c}T_{b}^{*} \\
\text { Myr }\end{array}$ \\
\hline PER OB1 & - & $9.5_{-1.7}^{+2.5}$ & - & $8.9_{-3.4}^{+7.6}$ \\
CAR OB1 & $8.3_{-2.2}^{+4.7}$ & $8.8_{-2.2}^{+4.7}$ & $<10$ & $7.4_{-5.1}^{+1.1}$ \\
SGR OB1 & - & $4.1_{-0.4}^{+0.5}$ & - & $3.3_{-3.3}^{+2.7}$ \\
\hline
\end{tabular}

Examination of Figure 6 and Figure 7 shows that the suspected expansion/compresssion of some OB-associations is based on the velocities of a few stars, sometimes only one. For example, excluding only one of 10-11 stars from the associations Mon OB2 and NGC 2439 (HD 46573 from Mon OB2 and HD 63423 from NGC 2439) reduces the absolute values of the parameters of expansion/compresssion to the noise level. A similar situation is observed in Cen OB1, which demonstrates compression along l-coordinate, $p_{l}=-87 \pm 33$, at $P>2.5 \sigma$ confidence level. However, excluding four of 27 stars (HD 115363, HD 114340, HD 110946 and HD 116119) decreases $\left|p_{l}\right|$ to the noise level, $p_{l}=-24 \pm 20 \mathrm{~km} \mathrm{~s}^{-1} \mathrm{kpc}^{-1}$.

We excluded from our consideration associations Mon OB2, NGC 2439, and Cen OB1 as having unreliable parameters of expansion/compression. The other thee associations (Sgr OB1, Per OB1, and Car OB1) with $\tilde{u}_{l}$ and $\tilde{u}_{b}$, determined at $P>2.5$ confidence level demonstrate expansion.

Figure 8 illustrates the expansion of the three OBassociations considered. It shows the linear increase of proper-motion components, $\mu_{l}$ or $\mu_{b}$, with the corresponding coordinate, $l$ or $b$, what suggests the expansion in the chosen direction (a decrease would indicate contraction). It can be seen that in all cases considered the spurious expansion determined by Eq. 34 is conspicuously smaller than the observed expansion.

Generally, the motion of OB-association as a whole in the sky plane with the tangential velocity $V_{t}$ also affects the velocity distribution inside association by producing spurious compression in one part of association and spurious expansion in another part. The velocity of this spurious expansion/compression $e_{2}$ is determined by the following relation:

$e_{2}=V_{t} \frac{a^{2}}{2 r^{2}}$

with $V_{t}$ in the following form:

$V_{t}=4.74 r \cdot \sqrt{\mu_{l}^{2}+\mu_{b}^{2}}$,

where $\mu_{l}$ and $\mu_{b}$ are given in Table 1 . Formally, the velocity $e_{2}$ spuriously increases the velocity dispersion inside OBassociation, but for OB-associations considered, $e_{2}$ doesn't exceed $e_{2}<0.1 \mathrm{~km} \mathrm{~s}^{-1}$ and its contribution can be neglected.

\subsection{Kinematic ages of OB-associations}

We assume that all stars in an expanding OB-association started their motion at nearly the same time instant in the past and moved at nearly constant velocities; hence their 

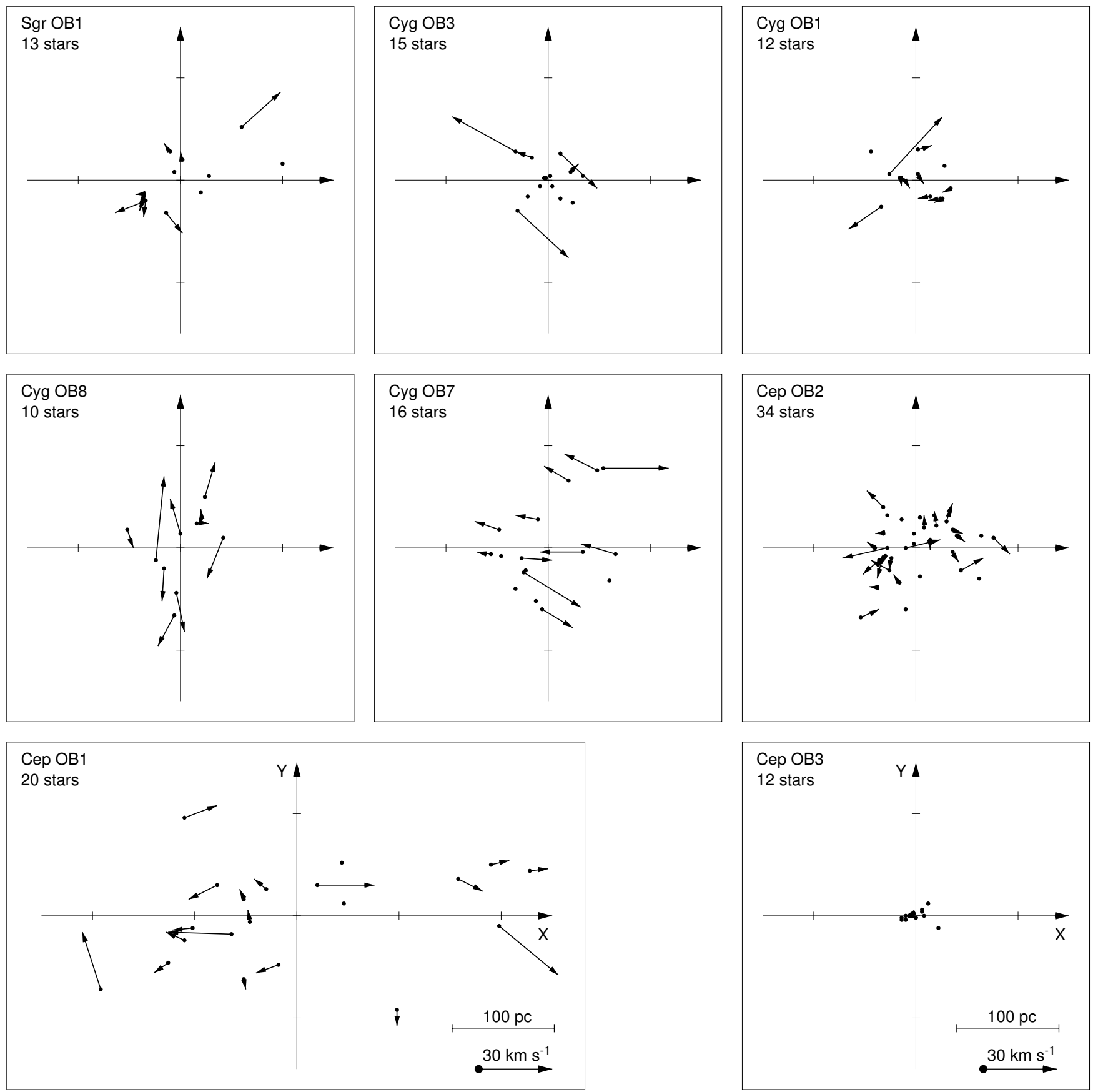

Figure 6. Distribution of the relative velocities $v_{l}^{\prime}$ and $v_{b}^{\prime}$ (Eq. 37 and 38) inside OB-associations with more than 10 TGAS stars. Stars with the relative velocities $\left|v_{l}^{\prime}\right|$ and $\left|v_{b}^{\prime}\right|$ smaller than $3 \mathrm{~km} \mathrm{~s}^{-1}$ are shown as black circles without any vector. The axes $X$ and $Y$ are directed toward increasing values of Galactic coordinates $l$ and $b$, respectively. All images are on the same scale. One tick corresponds to 100 pc. The Per OB1 association is not included in this panorama - we show it in a special image (Fig. 10). The Cep OB1 association is shown in a wider box because of its large extension in the l-direction. The velocity scale is shown in the bottom row.

distances from the center $\Delta x$ and $\Delta y$ are proportional to their observed velocities, $\Delta x=v_{l}^{\prime} \cdot t$ and $\Delta y=v_{b}^{\prime} \cdot t$. Though the distances and velocities are different for different stars, the time interval is supposed to be nearly the same for all stars in the OB-association considered. We estimate the socalled kinematic ages of expanding OB-associations by the formula:

$T_{l}=\left(p_{l} \cdot f_{v}\right)^{-1}$,
$T_{b}=\left(p_{b} \cdot f_{v}\right)^{-1}$

where factor $f_{v}=3.16 / 3.09 \cdot 10^{-3}$ transforms velocities in units of $\mathrm{km} \mathrm{s}^{-1}$ into $\mathrm{kpc} \mathrm{Myr}^{-1}$. The errors in $T_{l}$ and $T_{b}$ are determined by errors in $p_{l}$ and $p_{b}$, respectively.

Table 6 lists different estimates of the kinematic expansion ages of the three OB-associations considered. We can see that the $T_{l}$ and $T_{b}$ ages lie in the interval 4-10 Myr.

Here we ignore the epicyclic motions, which, through 

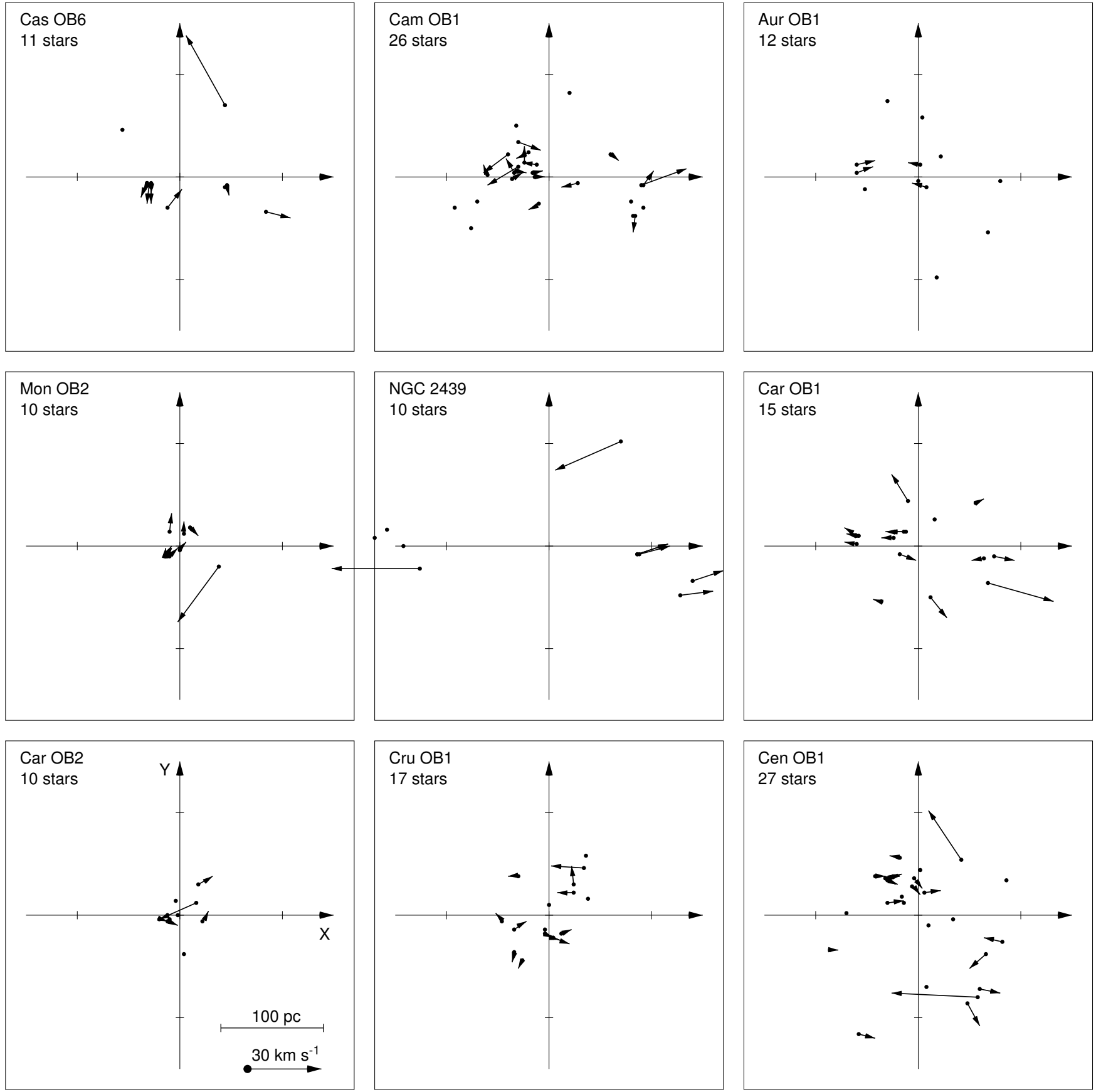

Figure 7. Distribution of the relative velocities $v_{l}^{\prime}$ and $v_{b}^{\prime}$ (Eq. 37 and 38) inside OB-associations with more than 10 TGAS stars. See caption to Figure 6 for more details.

Coriolis forces, change the direction of residual velocities (those superimposed on the Galactic rotation). This concerns only velocities in the Galactic plane. The Galactic rotation curve is nearly flat, and hence the epicyclic frequency at the solar distance is $\kappa=\sqrt{2} \Omega_{0}$ or $43 \mathrm{~km} \mathrm{~s}^{-1}$ $\mathrm{kpc}^{-1}$, and one quarter of the epicyclic period amounts to 37 Myr. It is this time interval that takes the Coriolis force to change the direction of residual velocities by $90^{\circ}$. We can see that the average age of OB-stars, $\sim 15 \mathrm{Myr}$, amounts to $\sim 40$ percent of one quarter of the epicyclic period. However, epicyclic motions change the direction of expansion but they do not prevent it. So the value of $p_{l}$ is determined by some combination of the expansion in l-direction and that in the perpendicular direction - along the line of sight. Parameter $p_{l}$ should rather be viewed as characterizing the expansion in the Galactic plane. Here we do not consider the expansion along the line of sight because with TGAS data the accuracy of sky-plane velocity components is for the first time better than that of line-of-sight velocities.

There is also another method to determine the time in the past when OB-associations had minimal sizes (see also Brown, Dekker \& de Zeeuw 1997). We trace back the positions of stars in OB-association using their relative velocities $v_{l}^{\prime}$ and $v_{b}^{\prime}$ : 

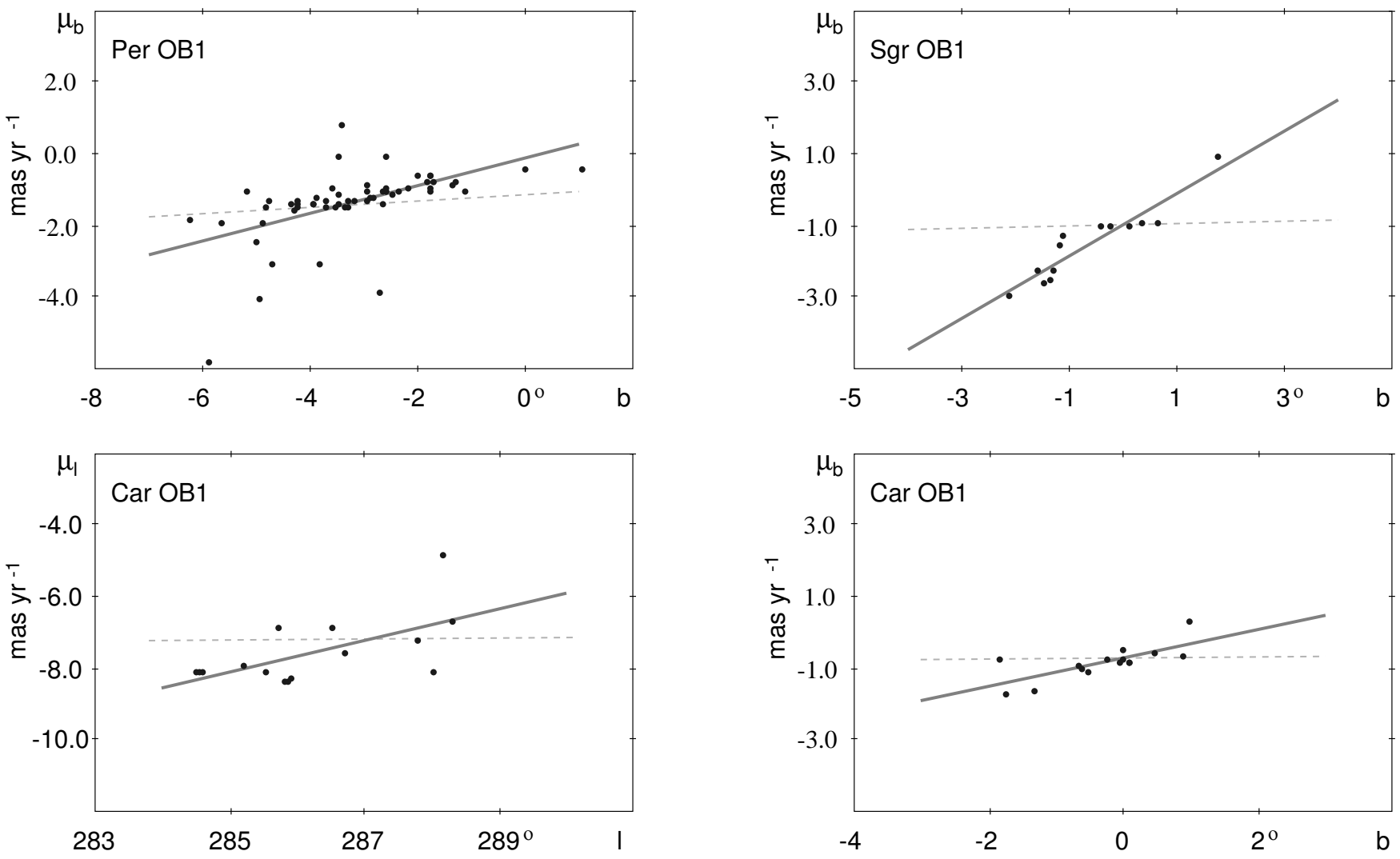

Figure 8. Dependence of the proper motions $\mu_{l}$ or $\mu_{b}$ on the corresponding Galactic coordinates, $l$ or $b$, calculated for TGAS stars in associations Per OB1, Sgr OB1 and Car OB1. The solid line indicates the observed linear dependence. The increase of proper-motion component along the corresponding coordinate indicates expansion in the chosen direction. The thin dashed line displays the contribution of spurious expansion due to line-of-sight velocity $V_{r}$ (Eq. 34). In all cases considered the spurious expansion is conspicuously smaller than the observed expansion.
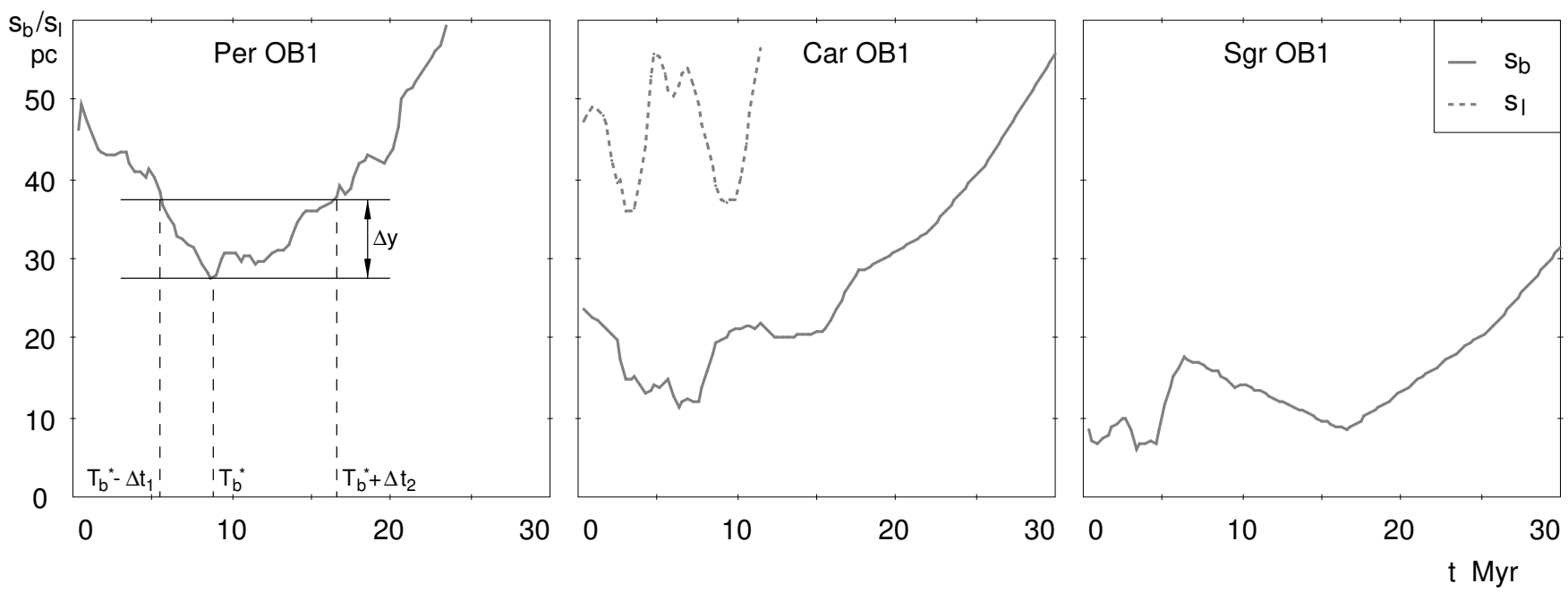

Figure 9. Dependence of the half-member size of OB-association in the b- or l-direction, $s_{b}$ or $s_{l}$, on the time $t$ in the past. Here $s_{b}$ and $s_{l}$ are the lengthes of the minimal intervals containing 50 percent of members of OB-associations, $n_{\mu}$, at the corresponding time moments. The positions of stars at each time instant were calculated by Eqs. 43 and 44 Only OB-associations with well-determined parameters of expansion are considered. We illustrate the method of deriving errors in kinematic ages, $\Delta t_{1}$ and $\Delta t_{2}$, with the example of the Per OB1 association: a horizontal line $y=s_{b}\left(T_{b}^{*}\right)+\Delta y$ intersects the curve $s_{b}(\mathrm{t})$ at two points with abscissae of $T_{b}^{*}-\Delta t_{1}$ and $T_{b}^{*}+\Delta t_{2}$, where $\Delta t_{1}$ and $\Delta t_{2}$ are error estimates on the left and right of the minimum. 
$x(t)=x_{0}-v_{l}^{\prime} t$,

$y(t)=y_{0}-v_{b}^{\prime} t$,

where $x_{0}$ and $y_{0}$ are the observed coordinates of TGAS stars with respect to the centre of OB-association, and compute the sizes of the most compact parts of OB-association at different time instants. The sizes, $s_{l}(\mathrm{t})$ and $s_{b}(\mathrm{t})$, containing 50 percent of TGAS stars of the corresponding OB-association are calculated in the following way. We sort the coordinates of association member stars, $x$ and $y$, in the ascending order and seek the subset $S=\{j+1, j+2 \ldots j+k\}$ containing $k=n_{\mu} / 2$ stars (where $n_{\mu}$ is the number of TGAS stars in OB-association) having the smallest span in the corresponding coordinate, $x_{j+k}-x_{j+1}$ and $y_{j+k}-y_{j+1}$. These minimal spans in the $\mathrm{X}$ - and $\mathrm{Y}$-direction give the values of $s_{l}(\mathrm{t})$ and $s_{b}(\mathrm{t})$, respectively. That is done to mitigate the effect of individual stars.

Figure 9 shows the dependence of $s_{b}$ and $s_{l}$ on the time $t$ in the past. We consider only OB-associations Per OB1, Car OB1 and Sgr OB1 with well-determined parameters $\tilde{u}_{l}$ or $\tilde{u}_{b}$. The curves $s_{b}(\mathrm{t})$ and $s_{l}(\mathrm{t})$ are often indented and some of them have several local minima, which are due to abrupt changes of the estimated size due to inclusion/exclusion of individual stars in the half-member minimal-span subset. We determine the kinematic expansion ages of OBassociations, $T_{b}^{*}$ and $T_{l}^{*}$, as the time instants $t$ in the past corresponding to the minimal values of $s_{b}$ and $s_{l}$, respectively (Fig. 9). However, in some cases we can give only an upper estimate for the kinematic age.

Table 6] lists the inferred $T_{l}^{*}$ and $T_{b}^{*}$ ages and their errors, which are calculated through the distances, $\Delta x$ and $\Delta y$, in the l- and b-direction which stars pass due to their errors in proper motions, $\varepsilon_{\mu l}$ and $\varepsilon_{\mu b}$, during the time intervals $T_{l}^{*}$ and $T_{b}^{*}$, respectively:

$\Delta x=2 \cdot 4.74 r \varepsilon_{\mu l} \cdot T_{l}^{*} f_{v}$,

$\Delta y=2 \cdot 4.74 r \varepsilon_{\mu b} \cdot T_{b}^{*} f_{v}$,

where $\varepsilon_{\mu l}$ and $\varepsilon_{\mu b}$ are in mas $\mathrm{yr}^{-1}$ but $\Delta x, \Delta y$ and $r$ are in kpc. Factor two reflects possible changes of the sizes of OBassociation at left and right sides of the distribution. The average errors in proper motions, $\varepsilon_{\mu l}$ and $\varepsilon_{\mu b}$, lie in the range 0.05-0.07 mas $\mathrm{yr}^{-1}$. The distances $\Delta x$ and $\Delta y$ increase with increasing the distance to the OB-association, $r$, and with increasing kinematic ages, $T_{l}^{*}$ or $T_{b}^{*}$. They have the following values: $\Delta y=10 \mathrm{pc}(\mathrm{Per} \mathrm{OB} 1), \Delta x \approx \Delta y=7-10 \mathrm{pc}$ (Car $\mathrm{OB} 1)$ and $\Delta y=4 \mathrm{pc}$ (Sgr OB1).

To transform errors in distances, $\Delta x$ and $\Delta y$, into errors in time intervals, $\Delta t_{1}$ and $\Delta t_{2}$, we use following expressions:

$s_{l}\left(T_{l}^{*}\right)+\Delta x=s_{l}\left(T_{l}^{*}-\Delta t_{1}\right)=s_{l}\left(T_{l}^{*}+\Delta t_{2}\right)$,

$s_{b}\left(T_{b}^{*}\right)+\Delta y=s_{b}\left(T_{b}^{*}-\Delta t_{1}\right)=s_{b}\left(T_{b}^{*}+\Delta t_{2}\right)$,

where $\Delta t_{1}$ and $\Delta t_{2}$ are the errors estimated on the left and right of the minimum on the curves $s_{l}(\mathrm{t})$ or $s_{b}(\mathrm{t})$. To illustrate this method we chose association Per OB1 and drew a horizontal line with the ordinate $y=s_{b}\left(T_{b}^{*}\right)+\Delta y$ which intersects the curve $s_{b}(\mathrm{t})$ at two points with abscissae of $T_{b}^{*}-\Delta t_{1}$ and $T_{b}^{*}+\Delta t_{2}$ (Fig. 9).

Table 6 shows that the kinematic ages of OBassociations obtained by two different methods, $T_{l}$ and $T_{l}^{*}$ or $T_{b}$ and $T_{b}^{*}$ agree with each other within the errors. The kinematic ages, $T_{l}, T_{l}^{*}, T_{b}$ and $T_{b}^{*}$, determined by both methods for three OB-associations considered lie in the range 3-10 Myr. These estimates do not exceed the main-sequence ages of O-B2 stars, $T_{s}<30$ Myr (Bressan et al. 2012).

\subsection{Minimal sizes of OB-associations in the past}

The second method is also interesting because it gives us the estimates of the minimal sizes of OB-associations corresponding to the time instants $T_{l}^{*}$ and $T_{b}^{*}$ (Fig. 9). The minimal sizes of the most compact parts of OB-associations containing 50 percent of star-members, $s_{l}\left(T_{l}^{*}\right)$ and $s_{b}\left(T_{b}^{*}\right)$, appear to be $27 \mathrm{pc}$ for Per OB1, 11-36 for Car OB1, and 6 pc for Sgr OB1.

Figure 9 shows that sizes of Per OB1 and Car OB1 in latitude directions, $s_{b}$, were nearly twice smaller in the past than nowadays. The formula for the virial mass (Eq. 20) includes the radius of the system $a$ referred to the epoch of star formation and we therefore should correct their virial masses $M_{v i r}$. Assuming that the contributions to $a^{2}$ from the coordinates $x$ and $y$ are nearly the same, we must reduce $M_{v i r}$ of Per OB1 and Car OB1 by the factor $(1+0.25) / 2)^{1 / 2}=0.79$ and upscale star-formation efficiency $\epsilon$ by a factor of 1.26 to the new values of 4.4 and 1.8 percent, respectively.

We can see that the minimal sizes of the Per OB1 and Car OB1 associations lie in the range $11-27$ pc and are consistent with diameters of giant molecular clouds, 10-80 pc. As for Sgr OB1, the situation is unclear here: we see too quick expansion in very small area. Moreover, it contains only 13 stars with known TGAS proper motions and errors

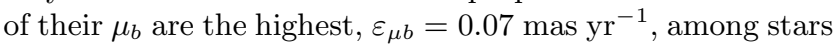
in the OB-associations considered.

Possibly, the quick expansion of Sgr OB1, as well as the large velocity dispersion in Cyg OB7 and Cyg OB8 have the same origin - larger errors of proper motions in fields rich in bright stars.

\subsection{Expansion of Per OB1}

Let us consider the expansion of the Per OB1 association in more detail (Fig. 10). It exhibits significant expansion along b-coordinates, $p_{b}=103 \pm 22 \mathrm{~km} \mathrm{~s}^{-1} \mathrm{kpc}^{-1}$ (Table 5). However, the expansion can be even faster if we tilt the coordinate axes $(X, Y)$ by the angle $\alpha=26^{\circ}$ with respect to the Galactic plane. In the new reference frame $\left(X^{\prime}, Y^{\prime}\right)$ the expansion parameter has the values of $p_{y}^{\prime}=136 \pm 25 \mathrm{kpc}^{-1}$ corresponding to the velocity $7.5 \mathrm{~km} \mathrm{~s}^{-1}$. Interestingly, the new axis $X^{\prime}$ is nearly parallel to the line connecting two rich open clusters NGC $869\left(l=134.63^{\circ}, b=-3.74^{\circ}\right)$ and NGC $884\left(l=135.07^{\circ}, b=-3.59^{\circ}\right)$ also known as the double cluster $h$ and $\chi$ Persei lying practically at the centre of OBassociation Per OB1. So the maximal expansion happens in the direction nearly perpendicular to the main plain of the double cluster.

We subdivide stars of Per OB1 into two groups: red supergiants of spectral type $\mathrm{K}$ and $\mathrm{M}$ (nine objects with TGAS proper motions) and stars of type O-B (49 objects). The ages of red supergiants must be less than 50 Myr while those of the main-sequence O-B2 stars must be less than 30 Myr (Bressan et al. 2012). However, we do not see difference in their kinematical behavior: both groups take part in the expansion along $1-$ and b-directions. The parameters 


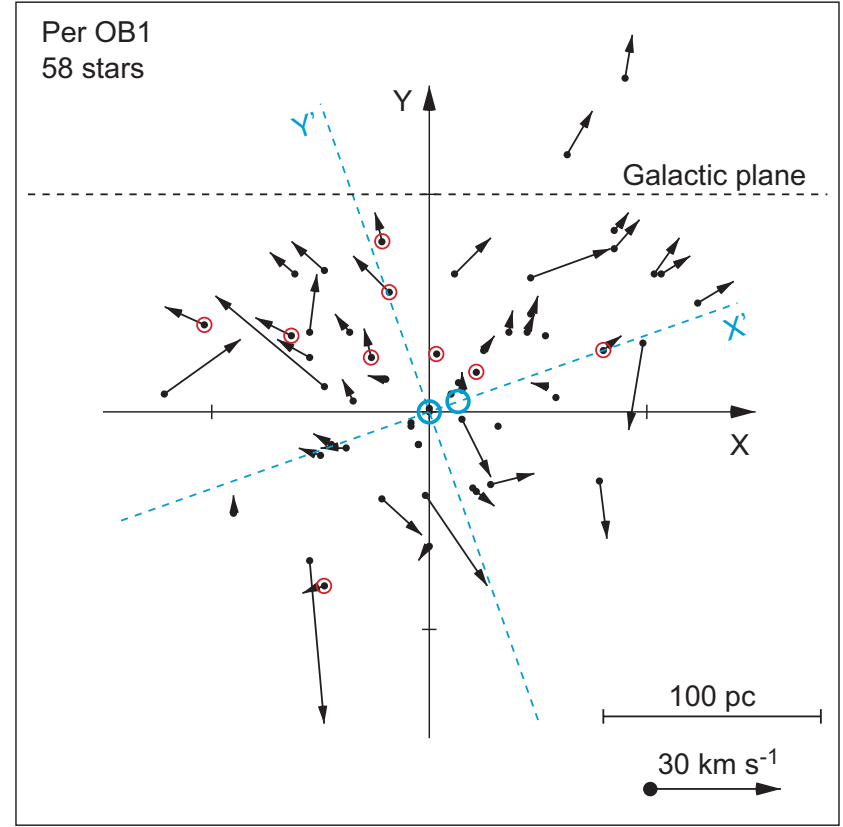

Figure 10. Distribution of relative velocities $v_{l}^{\prime}$ and $v_{b}^{\prime}$ in the Per OB1 association. Axes $X$ and $Y$ are directed toward increasing values of Galactic coordinates $l$ and $b$, respectively. Two open clusters NGC $869\left(l=134.63^{\circ}, b=-3.74^{\circ}\right)$ and NGC 884 $\left(l=135.07^{\circ}, b=-3.59^{\circ}\right)$ lying practically in the centre of the OB-association are shown as circles (colored blue in electronic edition). The line connecting them (the dashed line) is tilted by $20^{\circ}$ to the Galactic plane. The expansion of Per OB1 $(p=136 \pm 25$ $\left.\mathrm{km} \mathrm{s}^{-1} \mathrm{kpc}^{-1}\right)$ is the fastest in the direction nearly perpendicular to this line. The supergiants of type $\mathrm{K}$ and $\mathrm{M}$ are outlined by circles (colored red in electronic edition). The position of the Galactic plane is shown by the black dashed line.

of expansion obtained for red supergiants exclusively are $p_{l}=75 \pm 15$ and $p_{b}=61 \pm 14 \mathrm{~km} \mathrm{~s}^{-1} \mathrm{kpc}^{-1}$, while those derived for OB-stars only are $p_{l}=35 \pm 18$ and $p_{b}=109 \pm 26$ $\mathrm{km} \mathrm{s}^{-1} \mathrm{kpc}^{-1}$. These parameters are consistent within $1.2 \sigma$. Probably, red supergiants and OB stars observed in Per OB1 have very close ages and take part in the same expansion.

Cappa \& Herbstmeier (2000) notice that there is a lot of neutral hydrogen and no molecular gas in the vicinity of the Per OB1 association. They found a lot of HI bubbles surrounding massive WR and Of stars there.

Note that the evolutionary ages of clusters $h$ and $\chi$ Persei are comparable to those of stars in OB-associations, 10 Myr (Dias et al. 2002; Kharchenko et al. 2013) and the infered expansion age of the association. Possibly, the expansion of Per OB1 and formation of double stellar cluster $h$ and $\chi$ Persei in its centre have the same cause - the quick loss of gas in their parent giant molecular cloud.

As for double clusters, Kroupa, Aarseth \& Hurley (2001) find multiple clusters in some of their simulations of the formation of bound clusters inside the expanding OB-associations. Though their models are very compact, $\sim 10 \mathrm{pc}$, the physical mechanism considered can be universal. The authors explain the formation of double clusters in their models by gravitational interactions between neighbour stars, which cause the redistribution of the velocity dispersion from the radial direction into the azimuthal one. This mechanism can lead to the formation of a subsystem with non-zero angular momentum about the origin of the expanding flow.

\section{CONCLUSIONS}

We have studied the kinematics of OB-associations identified by Blaha \& Humphrevs (1989) using the stellar proper motions from the TGAS catalog (Michalik, Lindegren \& Hobbs 2015). The average difference in the median proper potions of OB-associations obtained with TGAS and Hipparcos data

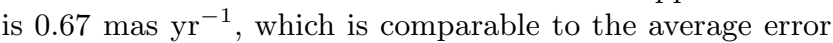

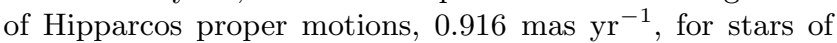
OB-associations.

Generally, the results based on TGAS and Hipparcos catalogs demonstrate a good agreement. The parameters of the Galactic rotation curve obtained with TGAS (Table 21) and Hipparcos (Mel'nik \& Dambis 2009) proper motions agree within the errors. The same can be said about residual velocities $\left(V_{r e s}=V_{o b s}-V_{r o t}-V_{a p}\right)$ of OB-associations in stellar-gas complexes identified by Efremov \& Sitnik (1988). The rms deviation of the velocities of OB-associations from the Galactic rotation curve amounts $\sigma_{0}=7.2-7.5 \mathrm{~km} \mathrm{~s}^{-1}$. This value is nearly the same whether computed with TGAS or Hipparcos data, suggesting that the residual velocities of $7-8 \mathrm{~km} \mathrm{~s}^{-1}$ are real and not an artifact.

When computed with TGAS data, the rms deviation of the velocities of OB- associations in the z-direction is $\sigma_{v z}=2.7 \mathrm{~km} \mathrm{~s}^{-1}$, which is considerably less than the scatter obtained with Hipparcos data, $\sigma_{v z}=5.0 \mathrm{~km} \mathrm{~s}^{-1}$.

We found a considerable decrease, by a factor of 0.4 , in the velocity dispersions inside 18 OB-associations containing more than 10 TGAS stars compared to the values derived with Hipparcos data. The average one-dimensional velocity dispersion inside OB-associations computed with TGAS catalog data is $\overline{\sigma_{v}}=3.9 \mathrm{~km} \mathrm{~s}^{-1}$.

The effective contribution from binary OB-stars into the velocity dispersion $\sigma_{v}$ inside OB-associations is $\sigma_{b}=1.2$ $\mathrm{km} \mathrm{s}^{-1}$.

The virial $M_{v i r}$ and stellar $M_{s t}$ masses of 18 OBassociations considered have the median values of $7.110^{5}$ and $9.010^{3} \mathrm{M}_{\odot}$, respectively. Generally, the virial mass exceeds the stellar mass by more than 70 times. This fact together with assumption that the volumes of OB-associations do not contain a lot of dense gas supports the conclusion that they are unbound objects.

The star-formation efficiency $\epsilon$ in OB-associations varies from 0.1 to 13.6 percent with the median of $\epsilon=2.1$ percent, which agrees with other estimates (Mvers et al. 1986; Evans et al. 2009; Garcia et al. 2014).

We determined the tidal radii of OB-associations under two assumptions: (1) OB-associations include neutral gas and its mass is proportional to the volume of the association, so that the present-day mass of the OB-association consists of masses of the gas and stellar components; (2) the masses of OB-associations are determined by their stellar component exclusively. In the first case, the median tidal radius appears to be $40 \mathrm{pc}$, suggesting that 27 percent of member stars of OB-associations are located outside their tidal radii. In the second case, the tidal radii range from 14 to $36 \mathrm{pc}$ with the median value of of $26 \mathrm{pc}$, implying that 39 percent of stars of OB-associations must lie outside their 
tidal radii. Thus, $\sim 1 / 3$ of stars of OB-associations must lie outside their tidal radius.

The high accuracy of TGAS proper motions allowed us to find the expansion inside the OB-associations Per OB1 and Car OB1 supporting the idea that they are unbound objects. The velocity of expansion amounts, on average, to $6.3 \mathrm{~km} \mathrm{~s}^{-1}$ (Table 5).

We corrected the observed specific velocities of expansion/compression, $u_{l}$ and $u_{b}$, for the effect of spurious expansion/compression caused by the motion of OB-association as a whole with the line-of-sight velocity $V_{r}$ (Eq. 34). Only cases with well-determined corrected velocities, $\tilde{u}_{l}$ and $\tilde{u}_{b}$, are considered (Table 5).

The kinematic ages of the expanding OB-associations Per OB1 and Car OB1 (time moments in the past when they had their minimal sizes) calculated by two methods are 7-10 Myr. The minimal diameter of the most compact parts of these OB-associations in the past lies in the range 11-27 pc which is comparable to the diameters of Galactic giant molecular clouds, $10-80$ pc Sanders, Scoville \& Solomon 1985).

We studied in more detail the expansion of the Per OB1 association. The direction of its fastest expansion $\left(Y^{\prime}\right.$ in Fig. (10) appears to be tilted by $26^{\circ}$ with respect to the b-direction and is nearly perpendicular to the plane determined by the positions of the two rich open clusters, $h$ and $\chi$ Persei, lying in the centre of the association. We found no significant difference between the parameters of expansion derived for OB-stars and red supergiants in this association.

\section{ACKNOWLEDGEMENTS}

We thank A.G.A. Brown for fruitful discussion. We thank A.S. Rastorguev and O.K. Sil'chenko for useful remarks and suggestions. This work has made use of data from the European Space Agency (ESA) mission Gaia (https://www.cosmos.esa.int/gaia), processed by the Gaia Data Processing and Analysis Consortium (DPAC, https://www. cosmos.esa.int/web/gaia/dpac/consortium). Funding for the DPAC has been provided by national institutions, in particular the institutions participating in the Gaia Multilateral Agreement. This research has made use of the VizieR catalogue access tool, CDS, Strasbourg, France. The original description of the VizieR service was published by Ochsenbein, Bauer \& Marcout (2000). Analysis of observational data was supported by Russian Scientific Foundation grant no. 14-22-00041.

\section{REFERENCES}

Aldoretta, E. J., et al., 2015, AJ, 149, 26

Ambartsumian, V. A., 1949, Soviet Astron. Zhurn., 26, 3

Barbier-Brossat, M., Figon, P., 2000, A\&AS, 142, 217

Baumgardt, H., Kroupa, P., 2007, MNRAS, 380, 1589

Blaauw, A., 1964, ARA\&A, 2, 213

Blaha, C., Humphreys, R. M., 1989, AJ, 98, 1598

Bobylev, V. V., Baikova, A. T., 2012, Astron. Lett., 38, 638

Boehle, A., et al., 2016, ApJ, 830, 17

Boily, C. M., Kroupa, P., 2003a, MNRAS, 338, 665
Boily, C. M., Kroupa, P. 2003b, MNRAS, 338, 673

Branham, R. L., 2017, Ap\&SS, 362, 29

Bressan, A., Marigo, P., Girardi, L., Salasnich, B., Dal Cero, C., Rubele, S., Nanni, A., 2012, MNRAS, 427, 127

Brown, A. G. A., Dekker, G., de Zeeuw, P. T., 1997, MNRAS, 285, 479

Cappa, C. E., Herbstmeier, U., 2000, AJ, 120, 1963

Colín, P., Vázquez-Semadeni, E., Gómez, G. C., 2013, MNRAS, 435, 1701

Dale, J. E., Ercolano, B., Bonnell, I. A., 2012 MNRAS, 427,2852

Dambis, A. K., Berdnikov, L. N., Kniazev, A. Y., Kravtsov, V. V., Rastorguev, A. S., Sefako, R., Vozyakova, O. V., 2013, MNRAS, 435, 3206

Dambis, A. K., Mel'nik, A. M., Rastorguev, A. S., 1995, Astron. Lett., 21, 291

Dambis, A. K., Mel'nik, A. M., Rastorguev, A. S., 2001, Astron. Lett., 27, 58

Dias, W. S., Alessi, B. S., Moitinho, A., Lépine, J. R. D. 2002, A\&A, 389, 871

Efremov, Yu. N., Sitnik, T. G., 1988, Soviet Astron. Lett., 14, 347

Elmegreen, B. G., 1983, MNRAS, 203, 1011

ESA 1997, The HIPPARCOS and TYCHO catalogues. Astrometric and photometric star catalogues derived from the ESA HIPPARCOS Space Astrometry Mission, ESA SP, 1200

Evans, N. J., et al., 2009, ApJS, 181, 321

Feast, M. W., Laney, C. D., Kinman, T. D., van Leeuwen, F., Whitelock, P. A., 2008, MNRAS, 386, 2115

Francis, Ch., Anderson, E., 2014, MNRAS, 441, 1105

Franco, J., Shore, S. N., Tenorio-Tagle, G., 1994, ApJ, 436, 795

Fujii, M. S., Portegies Zwart, S., 2011, Sci., 334, 1380

Gaia Collaboration, et al., 2016 A\&A, 595, A1

Gaia Collaboration, et al., 2016 A\&A, 595, A2

Garcia, P., Bronfman, L., Nyman, L.-A., Dame, T. M., Luna, A., 2014, ApJS, 212, 2

Garmany, C. D., Stencel, R. E., 1992, A\&AS, 94, 211

Glushkova, E. V., Dambis, A. K., Mel'nik, A. M., Rastorguev, A. S., 1998, A\&A, 329, 514

Goldreich, P., Tremaine, S., 1980, ApJ, 241, 425

Groenewegen, M. A. T., Udalski, A., Bono, G., 2008, A\&A, 481, 441

Grosbol, P., 2016, A\&A, 585, 141

Grossman, A. S., Graboske, H.C., Jr., 1971, ApJ, 164, 475

Hills, J. G., 1980, ApJ, 225, 986

Hog, E., et al., 2000, A\&A, 355, L27

Hohle, M. M., Neuhäuser, R., Schutz, B. F., 2010, Astron. Nachr., 999, 789

Humphreys, R. M, McElroy, D. B., 1984, ApJ, 284, 565

Kharchenko, N. V., Piskunov, A. E., Schilbach, E., Röser, S., Scholz, R.-D., 2013, A\&A, 558, 53

Kim, J.-G., Kim, W.-T., Ostriker, E. C., 2016, ApJ, 819, 137

Kroupa, P., 2002, Sci. 295, 82

Kroupa, P., Aarseth, S., Hurley, J., 2001, MNRAS, 321, 699

Krumholz, M. R., Matzner, C. D., McKee, C. F., 2006, ApJ, 653, 361

Larson, R. B., 1981, MNRAS, 194, 809

Lindegren, L., et al., 2016, A\&A, 595, A4 
McKee, C. F., 1989, ApJ, 345, 782

McKee, C. F., Ostriker, E. C., 2007, ARA\&A, 45, 565

Mac Low, M.-M., Klessen, R. S., 2004, Reviews of Modern Physics, 76, 125

Madsen, S., Dravins, D., Lindegren, L., 2002, A\&A, 381, 446

Mason, B. D., Gies, D. R., Hartkopf, W. I., Bagnuolo, W. G., ten Brummelaar, T., McAlister, H. A., 1998, AJ, 115,821

Mel'nik, A. M., Dambis, A. K., 2009, MNRAS, 400, 518

Mel'nik, A. M., Dambis, A. K., Rastorguev, A. S., 1999, Astron. Lett., 25, 518

Mel'nik, A. M., Dambis, A. K., Rastorguev, A. S., 2001, Astron. Lett., 27, 521

Mel'nik, A. M., Efremov, Yu. N., 1995, Astron. Lett., 21, 10

Mel'nik, A. M., Rautiainen, P., 2009, Astron. Lett., 35, 609

Mel'nik, A. M., Rautiainen, P., 2011, MNRAS, 418, 2508

Mel'nik, A. M., Rautiainen, P., Berdnikov, L. N., Dambis, A. K., Rastorguev, A. S., 2015, AN, 336, 70

Mel'nik, A. M., Rautiainen, P., Glushkova, E.V., Dambis, A. K., 2016, Ap\&SS, 361, 60

Michalik, D., Lindegren, L., Hobbs, D., 2015, A\&A, 574, 115

Myers, P. C., Dame, T. M., Thaddeus, P., Cohen, R. S., Silverberg, R. F., Dwek, E., Hauser, M. G., 1986, ApJ, 301, 398

Nikiforov, I. I., 2004, ASP Conf. Ser. Vol. 316, Astron. Soc. Pac., San Francisco, p. 199

Ochsenbein, F., Bauer, P., Marcout, J., 2000, A\&AS, 143, 23.

Press, W. H., Flannery, B. P., Teukolsky, S. A., Wetterling, W. T., Kriz, S., 1987, Numerical Recipes: The Art of Scientific Computing, Cambrige Univ. Press

Rastorguev, A. S., Pavlovskaya, E. D., Durlevich, O. V., Filippova, A. A., 1994, Astron. Lett., 20, 591

Rautiainen, P., Mel'nik, A. M., 2010, A\&A, 519, 70

Reid, M. J., et al., 2009a, ApJ, 700, 137

Reid, M. J., Menten, K. M., Zheng, X. W., Brunthaler, A., Xu, Y., 2009b, ApJ, 705, 1548

Sana, H., 2017, IAU Symposium 329, in press, arXiv170301608

Sanders, D. B, Scoville, N. Z., Solomon, P. M., 1985, ApJ, 289,373

Sitnik, T. G., Mel'nik, A. M., 1996, Astron. Lett., 22, 422

Solomon, P. M., Rivolo, A. R, 1989, ApJ, 339, 919

Solomon, P. M., Rivolo, A. R., Barrett, J., Yahil, A., 1987, ApJ, 319, 730

Stark, A. A., Blitz, L., 1978, ApJ, 225, 15

Tutukov, A. V., 1978, A\&A, 70, 57

Vine, S. G., Bonnell, I. A., 2003, MNRAS, 342, 314

de Zeeuw P. T., Hoogerwerf R., de Bruijne J. H. J., Brown, A. G. A., Blaauw, A., 1999, AJ, 117, 354

Zinnecker, H., Yorke, H. W., 2007, ARA\&A, 45, 481 